\title{
GRASSROOT DEMOCRACY: \\ DAS ATHENISCHE EXPERIMENT
}

\author{
RALPH HÄUSSLER
}

Universität Osnabrück

Zusammenfassung: Im Zentrum dieser Arbeit steht der Gegensatz von Theorie und Praxis in der athenischen Demokratie im Bild des aktuellen Forschungsstandes. Es gilt zu evaluieren, ob die athenische Politik von Demagogen oder einem ungebändigten Demos beherrscht wird oder ob wir es mit einem stabilen System zu tun haben, in dem die Souveränität des Demos über viele Generationen systematisch ausgebaut wurde. Wie konnte der Einzelne durch aktive Teilnahme diese "grassroot democracy“ erleben und selbst gestalten? Schließlich soll die Frage gestellt werden, welche Lehren wir für unser eigenes Demokratieverständnis ziehen können, vor allem in Bezug auf aktive Teilnahme, Synergieeffekte in der Meinungsbildung, sowie Auslosung und Rotation von Ämtern.

Schlagworte: Antike und moderne Demokratie, Athen, Kleisthenes.

ABSTRACT: At the centre of this paper is a re-evaluation of the theory and praxis in Athenian democracy in the light of recent research. Were Athenian politics controlled by demagogues or by an untamed demos? Or are we dealing with a system that systematically extended the demos's sovereignty from generation to generation? How could the individual participate actively in this democracy, how could he experience and shape it? Finally, the question arises which lessons can be learned for our own notion of democracy, especially in relation to people's active participation, the synergies in shaping public opinion, as well as drawing by lot and rotation of office holders.

Keywords: Ancient and modern Democracy, Athens, Cleisthenes. 


\section{EINLEITUNG ${ }^{1}$}

(...) today, behind a smoke-screen of careful distancing and qualifications, the general feeling is that Athens is ultimately an ideal city, which we moderns must admire even if we can no longer aspire to such simplicity and perfection. ${ }^{2}$

$\mathrm{D}^{\mathrm{a}}$ as Konzept der Demokratie ist eine der erstaunlichsten Leistungen des antiken Griechenland - insbesondere Athens - und die Grundlage für viele weitere gesellschaftliche Entwicklungen der klassischen Epoche, die Athen zum intellektuellen Zentrum machten. ${ }^{3}$ In der Antike hatte die griechische Demokratie jedoch meist einen eher schlechten Ruf: Aristoteles (384-322) forderte die Herrschaft der Besten (aristoi) und nicht die der "Masse" und im 2. Jh. schuf Polybios den Begriff der ochlokratie, der „Pöbelherrschaft" ${ }^{4}$ bei der es nicht mehr um das Gemeinwohl, sondern um persönliche Eigeninteressen gehe. Doch der aktuelle Forschungsstand zeigt ein ganz anderes Bild: Wie schon Claude Mossé argumentiert hat, war die athenische Demokratie „nicht das von Philosophen angeprangerte radikale und exzessive Regime, sondern ein weises und stabiles Regime“, das die Souveränität des dēmos (Volk) respektierte. ${ }^{5}$ Diese Souveränität wurde über viele Generationen konsequent ausgebaut, denn im Gegensatz zu modernen Demokratien handelte es sich um eine Basisdemokratie. Dabei erhielten weite Teile der Bürgerschaft, gleich welchen gesellschaftlichen Status', einen Anteil an der Macht. Gerade in heutigen bisweilen von Politikverdrossenheit geprägten Gesellschaften, die von Funktionärseliten, Bürokraten, Lobbyisten und Großkonzernen beherrscht erscheinen, soll diese Studie nicht nur das Demokratieverständnis und seine Entwicklung in Athen neu überdenken, sondern auch zum Nachdenken über die aktuelle politische Kultur anregen.

Grundlage der demokratischen Staatsform ist die Freiheit (eleutheria). Zur Freiheit gehört, dass man abwechselnd regiert und regiert wird. (...) Wo diese Gerechtigkeit herrscht, da muss die Menge (plethos) Herr (kyrios) sein, und was die Mehrzahl billigt, dass muss das Gültige und das Gerechte sein. (...) So sind dann in den Demokratien die Armen mächtiger als die Reichen. Denn sie sind zahlreicher, und maßgebend ist die Meinung der Mehrzahl. (...) Ein anderes (Zeichen der Demokratie) ist es, dass man leben kann wie man will (...). Von daher kommt, dass man sich nicht regieren lässt, am besten von überhaupt niemanden, oder dann doch nur abwechslungsweise. Auch dies trägt zur Freiheit im Sinne der Gleichheit bei. ${ }^{6}$

1. Für einen Überblick zur Demokratie, siehe z.B. Bleicken; STAHL, Klassische Zeit. Abkürzungen nach Kleiner Pauly. Alle Daten v. Chr. Ich bin Prof. Dr. Christiane Kunst und Dr. Achim Rost für ihre Kommentare und konstruktive Kritik sehr zum Dank verpflichtet.

2. Murray, Animals.

3. So schon J. Winkelmann, Geschichte der Kunst des Altertums (Dresden 1764) 1.4, 130-33.

4. Pol. $6,4,6 ; 6,4,10 ; 6,57,9$.

5. Mossé, Political Myth, 243.

6. Aristot. pol. 6, 1317a 40 - b 17. 
So beschreibt Aristoteles in seiner Politik die Grundlagen der Demokratie, die größtenteils auch heute noch gültig sind: die Entscheidung der Mehrzahl ist ausschlaggebend, das Gleichheitsprinzip, insbesondere die Rechtsgleichheit (isonomia), die Redefreiheit (isegoria), sowie die Freiheit, dass jeder Bürger leben kann, wie er will. Angesprochen sind damit die Grundrechte (sog. exousia „Macht ${ }^{\text {“7) }}$ ) für die ausgebildete Demokratie. Erklärung bedarf der Hinweis, dass man sich nicht regieren lässt bzw. „abwechselnd regiert und regiert wird“. ${ }^{8}$ Das bedeutet, dass jeder männliche Bürger nicht nur theoretisch das Recht hat, am demokratischen Prozess teilzunehmen, sondern viele auch tatsächlich an der Regierung teilgenommen haben bzw. teilnehmen mussten, insbesondere im Rat (boulé) und den Gerichtshöfen (dikastēria). Wichtiges Merkmal der athenischen Gesellschaft jener Zeit ist die kritische Selbstreflexion, wie sie zum Beispiel in den politischen Komödien des Aristophanes zum Ausdruck kommt, sowie in der Philosophie, insbesondere im Sokratismus: ${ }^{9}$ Redefreiheit und die Herausforderungen der Demokratie ermöglichen, dass man über die idealen Herrschaftsformen, die menschliche Natur und utopische Gesellschaftsformen ebenso nachdenken konnte, wie die Existenz der Götter anzweifeln - und das sogar in aller Öffentlichkeit, wie der systematische, beinahe blasphemische Angriff auf zeitgenössige religiöse Vorstellungen in Aristophanes" Komödie „Die Wolken“ zeigt. $^{10}$

Wie wir sehen werden, ist die griechische Demokratie das Ergebnis verschiedener Langzeitentwicklungen ab dem 8. Jh. v. Chr. mit einigen essentiellen Umbruchphasen, die wir mit Solon, Kleisthenes, Ephialtes und Perikles assoziieren. ${ }^{11}$ Erst durch die tatsächliche Ausübung der Macht durch die Mehrheit des dèmos entstand im Laufe des 5. Jh. v. Chr. ein demokratisches Bewusstsein. Wir erkennen eine ständige Weiterentwicklung der demokratischen Institutionen und einer Absicherung der Volkssouveränität. Doch wie sieht es in der Praxis aus? Ist der dēmos nur ein Spielball der Politiker? Wird er von Demagogen manipuliert und beherrscht? Oder handelt es sich um eine Herrschaft des „Pöbels“, wie uns die meist im 4. Jh. von Eliten verfassten und deshalb anti-demokratischen Schriften glauben lassen, insbesondere Aristoteles, Plato, Pseudo-Xenophon und Isokrates? ${ }^{12}$ Sind deshalb Fehlentscheidungen des dèmos an der Tagesordnung - vor allem auch wegen der vermeintlichen Inkompetenz von Ratsmitgliedern, Richtern und Volksversammlung? Oder können wir noch heute etwas von Athens demokratischer Kultur lernen?

7. Nikias, in Thuk. 7, 69, 2; Plato Resp. 557c; Isokrates 12, 131.

8. Cf. auch Euripides, Hiketiden, insbes. v. 406f.

9. Zur Entwicklung der politischen Theorie als Antwort auf die gesellschaftlichen Fragen und die politische Kultur, welche durch die Demokratie erst ermöglicht wurden, cf. FARrar, Democratic Thinking und MEIER, Discovery of Politics.

10. Aristoph. Nub. Cf. MacDowell, Aristophanes; Dover, Clouds.

11. Cf. z.B. RAAfLAUb, Entdeckung; ID., Einleitung und Bilanz.

12. Sind unsere Quellen wirklich so anti-demokratisch? Siehe dazu RAAFLAUB, Contemporary Perceptions für Verteidiger der Demokratie, z.B. Philosophen (Demokritos, Protagoras), Dramen-Schreiber (Aischylos, Euripiedes), Historiker (Herodot). 


\section{AUF DEM WEG ZUR DEMOKRATISCHEN BÜRGERGEMEINDE}

Die Ausbildung der athenischen Demokratie war ein langer Prozess. Die gesellschaftlichen Entwicklungen des 8.-6. Jh., die hier kurz angerissen werden sollen, waren eine wichtige Prämisse. Entscheidend ist das sich in dieser Epoche herausbildende Grundverständnis einer polis, nicht als Stadt sondern als Bürgergemeinde, die aus der Gesamtheit der Bürger besteht. ${ }^{13}$ Die Ausbildung der Hopliten als schwerbewaffnete Infanterie im 7. Jh., welche die Kavallerie der Adeligen ablöst, ist ebenfalls ein wichtiger Schritt: ${ }^{14}$ Es entsteht ein neues Gruppenbewusstsein, Spartas homoioi („Gleiche“) bzw. Athens Zensusklasse der zeugitai, bestehend aus denjenigen, welche die Bürgergemeinde verteidigen und die damit ein Anrecht auf politische Mitbestimmung haben. Die frühen Heeresversammlungen könnte man als den ersten Schritt in Richtung der demokratischen Volksversammlung sehen. Tatsächlich war es auch in Sparta, dem Hoplitenstaat par excellence, wo wir die Grundlagen demokratischer Institutionen finden: Spätestens um 600 gab es hier regelmäßig tagende "Volksversammlungen" mit probouleutischer (d.h. vorberatender) Funktion (Rat) in einer Gesellschaft, die das Gleichheitsprinzip der Hoplitengesellschaft schätzte. Doch entwickelte sich Sparta schließlich zu einem quasi „totalitären“ Regime. ${ }^{15}$

Ein weiterer, nicht zu unterschätzender Faktorist dieKolonisationsbewegung im 8.-6. Jh.: Griechische poleis gründeten in der gesamten Mittelmeerwelt Kolonien (apoikiai), bei denen alle Kolonisten theoretisch die selben Startchancen hatten. ${ }^{16}$ Die Idee des Gleichheitsprinzips hatte auch Einfluss auf das „Mutterland“. Kolonisation und der damit stetig wachsende Handel führten aber auch zu sozialen Umwälzungen: Während Unterschiede zwischen Reichen und Armen in den griechischen poleis immer größer werden, entstehen auch neue wirtschaftlich bedeutende soziale Gruppen, wie Handwerker und Händler. Dies hat extreme Konflikte zwischen Geburtsadel und den militärischen und wirtschaftlichen Eliten zur Folge. Gleichzeitig sind ab dem 7. Jh. immer wieder heftige inner-aristokratische Kämpfe (stasis) um soziale und politische Vorrangstellung belegt. Diese sozialen Spannungen können zur Tyrannis führen, also der Alleinherrschaft eines Volksführers, der die Adeligen ausschaltet und das „niedere“ Volk begünstigt. ${ }^{17}$

13. Cf. Murray, fr. Griechenland, 78-82; Osborne, Greece ${ }^{2}$, 128-130; für egalitarianism in archaischen poleis als Prämisse zur Demokratie, cf. RoBinson, First Democracies, 65-73, auch mit Diskussion zu MORRIS‘ archäologischen Befund für egalitarianism (Burial and Ancient Society, Cambridge 1987).

14. Cf. z.B. RaAflaub/Wallace, People's Power, 34-41.

15. Plut. Lykurg 6; Hornblower, Creation, 1; RaAflaub/Wallace, People's Power, 36-41.

16. Zur Gleichheit in den Kolonien, cf. Fitzjohn, Equality. Osborne, Greece ${ }^{2}$, 220-230.

17. Osborne, Greece ${ }^{2}$, 180-185; Berve, Tyrannis; Murray, fr. Griechenland, 175-201. 


\subsection{Solon - Mythos und Realität}

In dieser umwälzenden gesellschaftlichen Entwicklung, in der vorstaatliche Verwandtschafts- und Gefolgsschaftsorganisationen zunehmend durch politische und territoriale Organisationsformen ersetzt wurden, spielt Solon (ca. 638558 ) in Athen als Gesetzgeber eine entscheidende Rolle. ${ }^{18}$ Reichtum und Macht waren sehr ungleich verteilt. Aristoteles schreibt, dass das Volk „wenigen sklavisch untertan war (douleuein)" und deshalb „einen Aufstand gegen die Adeligen entfesselte". ${ }^{19}$ Solon - 594/3 von beiden Parteien zum Schiedsmann (diallaktés) und archōn (wörtlich „Herrscher") gewählt - schuf wichtige Grundlagen gegen die Willkür der Adeligen und die Ausbeutung der Bürger: Gesetze, Freiheit, Selbstverantwortung, Rechtsgleichheit und politische Institutionen, wie den Rat der 400, die Volksversammlung und die Volksgerichte (heliaia) (die beiden letzten waren der untersten Zensusklasse, den Theten, zugänglich): ${ }^{20}$

Dadurch, dass das Volk (dèmos) Herr (kýrios) über die Abstimmung ist, wird es auch Herr über das Staatswesen (politeías). ${ }^{21}$

Die Einführung der Rechenschaftspflicht der Beamten gegenüber den Bürgern und die Appellationmöglichkeit des Einzelnen gegen ihre Entscheidungen galten in der Aristoteles zugeschriebenen Schrift Athenaion Politeia („Der Staat der Athener“) des 4. Jh. als „im höchsten Maße demokratisch".22

Neben Fragmenten aus Solons eigenen Gedichten sind unsere Hauptquellen Aristoteles' Athenaion Politeia und Plutarchs Solon-Biographie, die aus einer Zeit stammen, als Solon zum Begründer der Demokratie "verklärt" worden war. Wie schon Claude Mossé gezeigt hat, ${ }^{23}$ gab es zur Zeit der völlig ausgestalteten Demokratie bei Athens Eliten den Wunsch zu einer fiktiven patrios politeia, ${ }^{24}$ der altüberkommenen „Verfassung der Vorväter“ zurückzukehren, in der alle Bürger zwar Gleichheit vor dem Gesetz hatten, aber nur die obersten Bevölkerungsschichten politisch aktiv waren. Aus Aristoteles' Beschreibung wird deutlich, dass Solons Taten aus der Perspektive des 4. Jh. gesehen wurden: Bereits die Terminologie ist typisch für Aristoteles' Zeit, wie beispielweise der Konflikt zwischen Armen (penetes) und Reichen (plousioi). ${ }^{25}$ Es gilt kritisch zu

18. Zu Solon cf. Stahl, Archaische Zeit, 176-251; Wallace, Revolutions, 59-75; zu Solons Zensusklassen, cf. RAAFLAUB, Breakthrough, 128-132.

19. Ath.Pol. 5,1. Cf. WAllace, Revolutions, 57-58.

20. Ath. Pol. 7, 3.

21. Ath. Pol. 9, 1; Cf. Hornblower, Creation, 5-6.

22. Ath. Pol. 9-10.

23. Mossé, Political Myth.

24. Hansen, Age of Demosthenes, 296-7: z.B. Ath.Pol. 29, 3; 34, 3. Nach 411/404 unmissverständlicher Bezug zur Demokratie, siehe Arist. Pol. 1273b38: patrios demokratia.

25. Auch Aristoteles' Erklärung der seisachtheia als Abschaffung der Schulden (chreon apokope) entspricht der Politik des 4. Jh.: siehe Mossé, Political Myth, 245. 
hinterfragen, was Solon wirklich getan hat. In seinen Gedichten erkennen wir Solons Selbstreflexion zu seiner eigenen Politik: ${ }^{26}$

Ich gewährte dem Volke so viel Gewalt, wie ihm zukommt,

Nahm seiner Würde nichts weg,

Fügte auch keine hinzu.

Wiederum ließ ich nicht zu, dass die reichen und mächtigen Herren

Mehr sich nähmen als das, was ihnen rechtens gebührt.

Also bewehrte mit starkem Schild ich beide Parteien,

Dass nicht wieder das Recht eine die andre bedrückt. ${ }^{27}$

Solons Ziel war die eunomia - die "gute, wohlgefügte Ordnung“ - , die als Reaktion auf eine gesellschaftliche Krise, nämlich die Ausbeutung der unteren Schichten durch eine "gewinnsüchtige Elite“, ausgelöst wurde. ${ }^{28}$ Ein Grundprinzip der eunomia ist die isonomia, die Gleichbehandlung aller Bürger vor dem Gesetz, welche willkürliche Entscheidungen der Elite beenden soll. ${ }^{29}$

Solons Hauptsorge betraf die Schuldknechtschaft, ${ }^{30}$ durch welche ein großer Teil der athenischen Einwohner in eine dauerhafte Abhängigkeit geraten war: „Wenn sie nicht ihre Pachtgelder ablieferten, gerieten sie selbst und ihre Kinder in Schuldknechtschaft“" ${ }^{31}$ Aristoteles beschreibt „die sklavische Abhängigkeit" all jener, die ihre Zugehörigkeit zur Gemeinde verloren haben: „denn sie hatten an nichts mehr Anteil““ ${ }^{32}$ Solons Lösung war die seisachtheia („Schuldenabschüttlung“), wodurch die hektemoroi, die Schuldknechte, befreit wurden und die persönliche Haftung für athenische Bürger abgeschafft wurde. ${ }^{33}$ Dies stieß bei den Adeligen auf Widerstand; allerdings konnte Solon auch die unteren Bevölkerungsschichten nicht zufriedenstellen, denn er lehnte andererseits auch eine egalitäre Landverteilung ab, wie sie dem spartanischen Gesetzgeber Lykurg zugeschrieben wird. ${ }^{34}$ Solon wollte den bestmöglichen Kompromiss und sah sich selbst „wie ein Grenzpfahl zwischen den Parteien“"35 und „wie ein gehetzter Wolf in der Mitte der Meute“.36

Diese wirtschaftliche Reform - die Schaffung zahlloser kleiner und mittlerer Bauernstellen, die zu mehr sozialer Gerechtigkeit führte - ist eine wichtige Grundlage für den Bürgerstaat und die Identität und Mentalität

26. Für die Anfänge der politischen Reflexion cf. RaAflaub, Poets; siehe auch RaAflaub, Legend or Personality.

27. Solon, zitiert nach Plut. Sol. 18.

28. Vgl. VAN WeEs, Gute Ordnung, 92.

29. Für Solons Gesetze: siehe RusChenbusCh, $\Sigma o \lambda \omega v o \varsigma$ Noroı.

30. Zur Schuldknechtschaft: siehe RaAflaub, Entdeckung, 54-65; Walter, An der Polis teilhaben, S. 196-199; Mossé, Political Myth, 243; HARris, New solution, 103-112.

31. Ath.Pol. 2, 2.

32. Walter, An der Polis teilhaben, S. 196-7; Ath.Pol. 2, 3.

33. Stahl/Walter, Athens, 145ff; Hornblower, Creation.

34. Für Lykurgs Landverteilung, cf. Plut. Lyk. 8, 1-4; Mossé, Political Myth, 244. Lykurg müsste vor 700 gewirkt haben, falls er eine reale Person war.

35. Solon F 25D.

36. Solon F 24D. 
seiner Mitglieder. ${ }^{37}$ Durch diese Aktion definierte Solon, was es heißt, Athener zu sein: statt ausschließliche Zugehörigkeit zu einer gentilen oder kultischen Gruppe und statt patriarchalischer Abhängigkeitsverhältnisse entstand so etwas wie Bürgerrecht und Staatlichkeit. ${ }^{38}$ Außerdem politisierte Solon die Bürger, die Anteil am Staat haben sollten, ${ }^{39}$ da er ihnen die Verantwortung für die Verteidigung dieser neuen Ordnung übergab, unter anderem um die Herrschaft des Adels durch Volksversammlung und Appellation einzuschränken. ${ }^{40}$

\subsection{Tyrannis $(546-510)$}

In der Zeit nach Solon kam es immer wieder zu Machtkämpfen, die in der Tyrannis - also in der nicht verfassungsmäßigen Alleinherrschaft - von Peisistratus (546-528/7) endeten. ${ }^{41}$ Ihm folgten Hippias und Hipparch; letzterer wurde 514 ermordet, während Hippias' Herrschaft, zuletzt zunehmend repressiv, 510 durch Sparta gestürzt wurde. ${ }^{42}$

Die lange Alleinherrschaft der Peisistradiden ist in vielerlei Hinsicht für die soziopolitische Entwicklung der Athener bedeutsam, da sie die Grundlagen für eine Bürgergemeinschaft vorangebracht hat, denn die Tyrannen "attempted to encourage a Panathenaic ideal". ${ }^{43}$ Die traditionellen Strukturen zwischen aristokratischen Familien und ihren „Klienten” wurden aufgebrochen, denn die Tyrannen unterstützten das politische Selbstbewusstsein der "normalen Bürger", die als Sponsoren von Festen und Bauprogrammen agieren konnten. ${ }^{44}$ Stahl und Walter schlussfolgern: „the tyranny (...) acted as a catalyst for the final breakthrough of the Athenian citizen-state".45

Das Ende der Peisistradiden war ein weiterer Stimulus. Hipparchs Ermordung durch Harmodios und Aristogeiton ${ }^{46}$ war zwar noch lange nicht der Beginn der „Demokratie”, wurde aber als Tyrannenmord im Folgenden glorifiziert und politisiert. Schon Kleisthenes soll 509 das erste Denkmal für die Tyrannenmörder - und damit das erste politische Denkmal überhaupt - in Auftrag gegeben haben. ${ }^{47}$ Die politische Perspektive nach der Tyrannis wäre eine erneute stasis gewesen, d.h. die Auseinandersetzung zweier sozialer Gruppen Athens, angeführt von Kleisthenes und Isagoras, wobei letzterer

37. STAHL, Archaische Zeit, 198-200.

38. Walter, An der Polis teilhaben, 199, n. 137; Hornblower, Creation; Stahl, ibd.

39. Z.B. Ath. Pol. 8,5 .

40. RAAFLAUB,Entdeckung, 45-57.

41. Zu den Peistratiden, cf. Lavelle, Sorrow and Pity.

42. Ath.Pol. 19, 1.

43. Frost, Aspects, p. 51.

44. OBer, Athenian Revolution, 267.

45. Stahl/Walter, Athens, 151.

46. Siehe dazu Ath.Pol. 18; Thuk. 6, 56-59.

47. Plin. NH 34, 17. 
von Sparta unterstützt wurde. Für stasis gab es im Allgemeinen keine friedlichen Lösungsmöglichkeiten, was somit wahrscheinlich zu einer neuen Alleinherrschaft geführt hätte, doch das wurde von der Mehrheit der Athener verhindert, die damit die Reformen des Kleisthenes initiierten. ${ }^{48}$

\section{EINE DEMOKRATISCHE REVOLUTION?}

\subsection{Kleisthenes' Reformen}

Die Athener waren stark geworden (nach dem Ende der Tyrannis). Das bürgerliche Recht des freien Wortes für alle ist eben in jeder Hinsicht etwas Wertvolles. ${ }^{49}$

Nach dem Ende der Tyrannis kam es zu den scheinbar umwälzenden Entwicklungen, welche zur Souveränität des dēmos führen sollten. Viele der Entscheidungen werden Kleisthenes zugeschrieben, der 509-507 die athenische Gesellschaft grundlegend revolutioniert haben soll: ${ }^{50}$ Für Herodot war er es, der "die Phylen und die Demokratie erschaffen hat". ${ }^{51}$ Doch nach 400 schwindet die Erinnerung an ihn: für Isokrates ${ }^{52}$ hat er lediglich Solons System wiederhergestellt. Josiah Ober ${ }^{53}$ bezweifelt die Rolle einer einzelnen Person, wie Kleisthenes, in dieser Entwicklung. Für ihn ist der Protagonist eindeutig der dèmos, der - vergleichbar mit der Französischen Revolution drei Tage lang 508/7 die Akropolis in einem spontanen, führerlosen Aufstand belagerte, in der sich Isagoras, der die boulé auflösen wollte, zusammen mit dem spartanischen König Kleomenes I. und dessen Truppen verschanzte. Kleisthenes selbst war zu jener Zeit im Exil. Unsere Quellen erwähnen keine politischen Führer oder Amtsträger, sondern sprechen von Massen (plēthos), Volk (dēmos) ${ }^{54}$ bzw. dem "Rest der Athener” (Athēnaiōn hoi loipoi). ${ }^{55} \mathrm{Als}$ Kleisthenes aus dem Exil zurückkehrte, nahm er sich dieser Bewegung an. Für Kurt Raaflaub ist dagegen die Epoche ab Ephialtes (462-1) bedeutender für den Beginn der Demokratie, da der athenische dèmos erst zu jener Zeit ein neues Selbstbewusstsein und den Begriff demokratia entwickelte. ${ }^{56}$ Dennoch werden unter Kleisthenes entscheidende Grundlagen für ein neues politisches Verständnis der athenischen Gesellschaft gelegt, welche, von den Perserkriegen und den wirtschaftlichen und militärischen Anforderungen katalysiert, eine

\footnotetext{
48. Warum die Mehrheit der Athener Kleisthenes unterstützten, cf. Stahl/Walter, Athens, 153.

49. Hdt. 5, 78 .

50. Zu Kleisthenes, cf. Ath.Pol. 20ff.

51. Hdt. 6, 131. Ebenso Ath. Pol. 29, 3.

52. Siehe z.B. Areopagiticus, 16

53. Ober, Athenian Revolution. ID., I Besieged.

54. Ath. Pol. 20,3.

55. Hdt. 5, 69, 2-70; 72-3; cf. STAhl/Walter, Athens, 154.

56. RaAflaub, Einleitung und Bilanz; ID., Introduction, 14f; ID., Breakthrough; Develin, Kleisthenes.
} 
Eigendynamik entwickeln wird, die innerhalb weniger Generationen zur Souveränität des dèmos in allen Entscheidungen führen wird.

Neben der isegoria (Redefreiheit) ist die Phylenreform eine wesentliche Grundlage für Kleisthenes' politische und gesellschaftliche Neuordnung Athens. Statt der bisherigen vier Phylen („Stämme“) schuf er zehn neue Phylen nach einer sehr abstrakt scheinenden Formel: Jede Phyle bestand aus drei Trittyen („Drittel“), je eine aus dem Gebiet der Stadt, der Küstenregion und dem Binnenland Attikas. Tatsächlich berichtet Aristoteles, dass Küsten-, Ebenen- und Bergbewohner in der Zeit vor der Tyrannis für verschiedene politische Richtungen standen:

Es gab drei Parteien: erstens die Küstenbewohner mit Megakles, dem Sohn des Alkmeon an der Spitze, die am ehesten eine gemäßigte Verfassung anstrebten; zweitens die in der Ebene Wohnenden, welche die Oligarchie wünschten. Ihr Anführer war Lykurg. Drittens die Bergbewohner mit ihrem Anführer Peisistratos, der am volksfreundlichsten zu sein schien. ${ }^{57}$

Zudem teilte Kleisthenes ganz Athen und Attika in rund 130 Demen (dēmos "Gemeinde") ein, ${ }^{58}$ die jeweils einem der dreißig Trittyen zugeordnet wurden. Ziel war die "Vermischung" der Bevölkerung, ${ }^{59}$ damit es keine Interessenkonflikte zwischen den verschiedenen Regionen Attikas gibt. Vor allem sollte die Bindung der einzelnen Phylen an eine adelige "Sippschaft" (geneá) beseitigt werden. Jede Phyle wurde somit zum Mikrokosmos der Bürgerschaft, in der die unterschiedlichen Interessen der Bewohner aus Stadt, Küste und Hinterland vertreten waren, wodurch ein neues kollektives Bewusstsein entstehen konnte. ${ }^{60}$

Die zehn Phylen bilden in der Folgezeit eine wesentliche Grundlage für alle staatlichen Institutionen in Athen, die sich gleichmäßig aus allen Phylen rekrutierten, wie die 6.000 Richter für die Gerichtshöfe. Das gilt ganz besonders für den unter Kleisthenes eingeführten Rat der Fünfhundert (boulé), in den jede Phyle jährlich 50 ausgeloste „Repräsentanten“ abordnen musste, eine genau festgelegte Anzahl für jede einzelne Deme, wodurch alle demoi aus ganz Attika gleichmäßig vertreten waren. Unter anderem zeigt dies, dass die athenische Bürgerschaft als eine Vielzahl sich selbst regierender lokaler Gesellschaften verstanden wurde. Man erkannte die Bedeutung komplexer zwischenmenschlicher Beziehungen und hat versucht, vom Potential des unterschiedlichen Wissens und den vielfältigen Erfahrungswerten der einzelnen Bürger aus den verschiedenen Demen zu profitieren. ${ }^{61}$ Die Demen

57. Ath.Pol. 13, 4

58. Ath. Pol. 21, 5: einige Demen sind Neuschöpfungen. 134 demoi sind im 4. Jh. belegt. Cf. WhiteHEAD 1986.

59. Ath.Pol. 21, 2-4.

60. Cf. Anderson, Athenian Experiment, 124-5.

61. So Ober, Democracy Today, 37. 
wurden in der Folge zudem die Basis der neuen Ordnung, da sie Funktionen der lokalen Selbstverwaltung übernahmen: Sie hatten jeweils ihre eigenen Beamten, insbesondere den demarch ${ }^{62}$ ihre eigenen Priester und Kulte, wie die ländlichen Dionysien, und sie führten die Bürgerlisten. ${ }^{63}$ In der "faceto-face society" auf Demen-Ebene war es somit möglich, Selbstverwaltung und das, was man im 5. Jh. Demokratie nennen würde, zu üben. Gleichzeitig blieben vorstaatliche Formen sozialer Organisation bestehen, insbesondere die von den adeligen genē dominierten Phratrien („Bruderschaften“), deren soziopolitischer Einfluss nun aber gering war. ${ }^{64}$

In dieser umwälzenden Zeit nach dem Sturz von Hippias war es das Ziel, stasis und tyrannis vorzubeugen. Das Konzept der Demokratie war unter Kleisthenes noch unbekannt. Dennoch schuf die neue "Verfassung" die Voraussetzungen für eine "grassroot democracy“, in der die Bürgerschaft durch Redefreiheit, Rechtsgleichheit, Volksversammlung, Rat und Gerichte die eigentliche Macht im Staat innehatte. In dieser Ordnung mussten sich Adelige, die politisch aktiv sein wollten, erst behaupten: Die ursprüngliche "Exekutivgewalt", die bei den Archonten lag, verlor zunehmend an Bedeutung (v. infra); durch die boulé war die Rolle des aus ex-Archonten bestehenden Areopags ohnedies stark eingeschränkt. Es entstand erneut eine Eigendynamik, die in der Folgezeit zum weiteren Ausbau der Macht des Volkes führen würde.

\subsection{Perserkriege (490-479)}

Hierbei sind die Perserkriege eine wichtige Etappe. Sie zeigen, dass Athen in der Lage war, eine effiziente Armee und Flotte aufzustellen, vielleicht auch als Ergebnis der Phylenreform, und dabei so erfolgreich war, dass es die Rolle als griechischer Hegemon von Sparta übernahm. Das Bündnis hellenischer poleis im Kampf gegen die Perser schuf zudem eine zunehmend „pangriechische" Identität. Im nachfolgenden attisch-delischen Seebund versetzten die Tributzahlungen der Bundesgenossen Athen in die Lage, den erheblichen Kostenaufwand der demokratischen Institutionen zu finanzieren. ${ }^{65}$ Ganz entscheidend ist die Rolle der Flotte, die Athen zur vorherrschenden Seemacht des 5. Jh. machen sollte: Themistokles überzeugte das Volk im Jahr 482, 200 Trieren mit dem aus den neu entdeckten Silberminen in Laureion gewonnen Geld zu bauen, was entscheidend zum Seesieg der Griechen über die Perser bei Salamis 480 führte. Mit rund 170 Ruderern pro Schiff brauchte man

62. Ath. Pol. 21, 5; siehe z.B. den Beschluss einer Deme aus dem 4. Jh.: Rhodes/Osborne, no. 63.

63. Für eine Studie zum ländlichen Attika, siehe JonEs, Rural Athens.

64. Die Phratrien sind nur bedingt ein Überbleibsel vorstaatlicher Clan-Strukturen, sondern haben sich den gesellschaftlichen Entwicklungen angepasst. Für LAMBERT, Phratries: „phratries reflected the democratic norm of the era".

65. Doch war die Demokratie (Diäten ab Perikles, v. infra) wirklich so teuer im Vergleich zu Athens ständiger militärischer Ausrüstung und seinen Bauprogrammen? 
34.000 Mann, die vor allem aus der untersten Bevölkerungsschicht Athens, den Theten, rekrutiert wurden, darüber hinaus auch Sklaven, die aber vorher frei gesetzt wurden. ${ }^{66}$ Dadurch erhielten auch die Theten politischen Einfluss:

Ebenso war die Masse der Seeleute die Ursache für den Sieg bei Salamis und darum auch der Hegemonie aufgrund der Herrschaft über das Meer, und so stärkte sie wieder die Demokratie. ${ }^{67}$

Selbst in der anti-demokratischen Schrift des Pseudo-Xenophons (425 ca. 354) wird somit die Herrschaft des dēmos legitimiert:

Zunächst muss ich es aussprechen, dass mit Recht daselbst die Armen und das Volk berechtigt sind, den Vorzug vor den Vornehmen und den Reichen zu haben, und zwar deshalb, weil nur das Volk es ist, dass die Schiffe treibt und dadurch der Stadt ihre Machtstellung verschafft (...), alle diese nur sind es, die der Stadt ihre Machtstellung verschaffen, wenigstens viel eher als das schwere Fußvolk und die Vornehmen und überhaupt die Edlen. Unter diesen Umständen erscheint es nur gerecht, dass allen bei der jetzt üblichen Losung wie bei der Wahl alle Ämter offenstehen und dass es jedem von den Bürgern, wer da will, freisteht, öffentlich zu reden. ${ }^{68}$

Die gesellschaftliche Entwicklung ging unaufhaltsam weiter und die Regeln der athenischen Verfassung passten sich dementsprechend an. Ein grundlegender Schritt - Kurt Raaflaubs "breakthrough of dēmokratia“69 - fand 462-461 unter Ephialtes statt, einer Persönlichkeit, über die wir nur wenig Informationen haben: 465 war er Stratege und 461 wurde er ermordet. ${ }^{70}$

Als aber die Volksmenge stärker wurde, gelangte Ephialtes, Sohn des Sophonides, der als unbestechlich und loyal gegenüber dem Staatswesen galt, an die Spitze des Volkes und richtete Angriffe gegen den Rat (auf den Areopag). Zunächst beseitigte er viele der Areopagiten, indem er gegen sie Prozesse wegen ihrer Amtsführung anstrengte. Dann, unter dem Archonten Konon [462/461], nahm er diesem Rat alle die hinzugekommenen Funktionen wieder, durch die er Wächter der Verfassung war, und übertrug die einen den Fünfhundert, die anderen dem Volk und den Gerichten. ${ }^{71}$

Nach den Perserkriegen "gewann der Rat auf dem Areopag wieder an Macht und verwaltete die Stadt" für 17 Jahre, wie uns Aristoteles berichtet. ${ }^{72}$

66. Thuk. VI 8; VIII 29, 2; Xen. Hellenica I 5, 3-7; cf. Ruschenbusch, Besatzung; Graham, Thucydides.

67. Aristot. pol. 1304a 21-24.

68. Ps.-Xen. Ath.pol. 1,2.

69. RaAflaub, Breakthrough.

70. Aristot. Pol. 1274a7; Ath.Pol. 25, 4; Diod. Sic. 11, 77, 6; Plut., Kimon 10,8; Plut. Perikles 7, 8; Pausanias $1,29,15$.

71. Ath.Pol. 25, 1-2.

72. Ath.Pol. 23, $1 ; 25,1$ 
Dafür gibt es aber keinen Beweis; ${ }^{73}$ zudem wurden die Archonten schon seit 487/6 aus den Phylen ausgelost, ${ }^{74}$ dennoch blieb der Areopag wohl "die letzte Bastion institutionalisierter aristokratischer Vorherrschaft" ${ }^{\text {"75 }}$ Die Aufgaben, die Ephialtes dem Areopag wegnahm, waren vermutlich die dokimasia - eine „Tauglichkeitsprüfung" für Amtsbewerber - und die eisangeliai, also seine Rolle als Aufsichtsbehörde über die Einhaltung der Gesetze und die Ahndung wegen Amtsvergehen. ${ }^{76}$ Je nach Aufgabengebiet wurden diese Aufgaben an boulé, ekklesia bzw. die Gerichtshöfe (dikastēria) übertragen. Dem Areopag blieben somit nur noch Sakralfunktion und Blutgerichtsbarkeit. Fünf Jahre nach Ephialtes' Ermordung wurde beschlossen, dass sich die Archonten auch aus der untersten Bevölkerungsschicht bzw. Zensusklasse, den Theten, rekrutieren konnten, ${ }^{77}$ womit die Rolle des Areopags als "oligarchisches" Gremium endgültig beendet war.

Unter Perikles (495/490-429) wurde die Verfassung dann noch

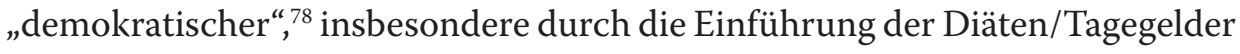
(misthós „Lohn/Sold“) 454-450, zuerst für die 6.000 Richter, aber auch für andere Amtsträger, insbesondere die Bouleuten. ${ }^{79}$ In der Antike wurden die Diäten oft kritisiert, da viele nur wegen der Bezahlung politisch aktiv gewesen seien, aber die Diäten waren eine Grundvoraussetzung für die Demokratie, denn ohne Bezahlung war es vielen Athenern unmöglich, im Rat, in den Gerichten oder an der Volksversammlung teilzunehmen. Dies widerspricht natürlich oligarchischen oder aristokratischen Verfassungsidealen, in denen die "Besten“ die Zeit und Muße haben sollten, über das Schicksal der polis zu debattieren. Erst die Diäten ermöglichen dem dèmos seine Souveränität auszuüben. Zudem war es eine Art der Umverteilung des Wohlstandes auf die untersten Bevölkerungsschichten, die so von Athens Reichtum und den Tributleistungen der athenischen Bündnispartner profitieren konnten.

73. Stahl, Klassische Zeit, 77-79.

74. Ath.Pol. 22, 5.

75. RAAFLAUB, Breakthrough, 114

76. Cf. Ath.Pol. 8, 4 (unter Solon); für die Rolle des Areopags, cf. RHodes, Athenian Revolution, 67-75; SChubert, Areopag; Stahl, Klassische Zeit, 77-79.

77. Ath.Pol. 26, 2.

78. Ath.Pol. 27, 1.

79. Ath.Pol. 27, 3. Zuerst von ca. 460-450 zwei Obolen (ab ca. 425 unter Kleon drei Obolen: Aristoph. Equ. 255) für die 6.000 Richter (Ath.Pol. 27, 3-5) und wohl auch für die Ratsherrn (5 Obolen für die Ratsherrn belegt für das 4. Jh.: Ath.Pol. 62,2); erst ab den 390er Jahren misthos für die Volksversammlung (Ath. Pol. 41,3; Aristoph. Eccl. 186-8, 289-311, 392). Nach der Tyrannis gab es auch Diäten für den Besuch der Volksversammlung (cf. Ath.Pol. 41, 3; 62, 2): anfangs 1 Obole, dann 2, schließlich 3 Obolen erhöht. Kleophon hat -vermutlich 411/410 - zwei Obolen misthos eingeführt, vielleicht für eine Anzahl von Amtsträgern, evtl. auch für den Theaterbesuch (Ath.Pol. 28, 3). Im Vergleich, der Sold für Ruderer der Flotte betrug drei Obolen (Thuk. 8, 45, 2); der Lohn für einen ungelernten Arbeiter am Erechtheion 409 v. Chr. dagegen mind. fünf Obolen (IG I2 372/274). Cf. HANSEN, Misthos; Bleicken, 233-241. 


\section{Demokratie: Theorie \& Praxis}

\subsection{Die Natur der athenischen Demokratie}

(...) alle Ämter werden aus allen besetzt, alle herrschen über jeden und jeder abwechslungsweise über alle. Ferner werden die Ämter durch Los besetzt, entweder alle oder doch jene, die nicht der Erfahrung und Kenntnisse bedürfen. (...) Keiner darf ein Amt zweimal bekleiden (...), abgesehen von den Kriegsämtern. Die Ämter sind alle kurzfristig (...). Richter können alle sein und können aus allen entnommen werden und richten über alles (...). Die Volksversammlung entscheidet über alles (...), die Behörden dagegen über nichts oder nur ganz weniges. ${ }^{80}$

Aristoteles beschreibt hier die Grundlagen der athenischen Demokratie: Annuität, Auslosung, Gleichheit und vor allem die Souveränität des dēmos durch Volksversammlung (ekklesia) und Gerichte (dikastēria), während boulé und Behörden der ekklesia nachgeordnet sind und ihr zuarbeiten. ${ }^{81}$ Die boulé bietet einen Querschnitt der Bevölkerung aus allen Demen Attikas: Sie bestand aus 50 Mitgliedern pro Phyle, wobei jeweils eine Phyle für ein Zehntel des Jahres (35/36 Tage) die Regierungsfunktion übernahm (sogenannte Prytanie) - die Reihenfolge wurde natürlich ausgelost, ebenso der täglich rotierende Vorsitz, ${ }^{82}$ um Einflussnahme auf die übrigen Ratsmitglieder zu minimieren. Die boulé hat zahlreiche Verwaltungsund Exekutivfunktionen, wie der Empfang von Botschaftern, Aufbewahrerin des Staatssiegels, etc., doch nach 411 wurde ihre Macht weiter beschnitten: „Der Rat (boulé) aber hatte früher die Befugnis, Geldstrafen, Verhaftungen und Todesstrafen zu verhängen... Das Volk aber entzog dem Rate (das Recht)“. ${ }^{83}$

Die wichtigste Rolle der boulé bestand darin, im Auftrag der ekklesia, Volksbeschlüsse vorzuformulieren (probouleumata), die dann der ekklesia vorgelegt wurden; das ermöglichte das Funktionieren der Volksversammlung, die den Wortlaut allerdings noch ändern konnte. Es war dem Volk nicht erlaubt über etwas abzustimmen, worüber der Rat nicht beraten hatte. Dadurch sollten spontane, willkürliche und nicht gesetzesmäßige Beschlüsse vermieden werden. Ein Quorum von 6.000 Leuten sollte zudem verhindern, dass Minderheiten politische Entscheidungen trafen. ${ }^{84}$ Das folgende ProxenieDekret $^{85}$ aus dem Jahr 408/7 zeigt den Ablauf eines Volksbeschlusses, die Rolle von Rat, Volksversammlung und dem einzelnen Bürger:

80. Aristot. pol. 1317b 19-30.

81. Überblick über die Verfassung, cf. STAHL, Klassische Zeit; BLEICKEN.

82. Ath.Pol. 44.

83. Ath.Pol. 45,1.

84. 6.000 Personen werden z.B. bei der Bürgerrechtsverleihung in Pseudo-Demosthenes, gegen Neaira 89f genannt; Thukydides' Bericht $(8,72,1)$ von 5.000 Teilnehmern in der Volksversammlung ist nicht vertrauenswürdig, alleine schon wegen der Kriegssituation des Jahres 411: siehe auch HANSEN, Ecclesia, 9 (für Thuk.); 26 (für Quorum von 6.000 und Größe des Versammlungsortes, die Pnyx).

85. Damit wurde ein proxenos geehrt, ein „Staatsgastfreund“, der die Interessen Athens in seiner Heimatgemeinde vertritt. 
Rat und Volk haben beschlossen, Antiochis hatte die Prytanie inne, Eukleides war Sekretär, Hierokles hatte den Vorsitz. Euktemon war Archon.

Dieitrephes stellte den Antrag. Da Oiniades von Palaiskiathos ein vortrefflicher Mann ist gegenüber der Stadt Athen (...) soll er als (...) Wohltäter der Athener eingetragen werden (...). Der Sekretär des Rats soll diesen Beschluss (psephisma) auf einen steineren Pfeiler aufschreiben und auf der Akropolis aufstellen lassen. Oiniades soll morgen auch zur Bewirtung in das Prytaneion eingeladen werden. Antichares stellt den Antrag: In allem übrigen besteht Übereinstimmung mit dem Rat, nur soll in der Beschlussvorlage „von Skiathos“ geändert werden, so dass „Oiniades von Palaiskiathos“ geschrieben steht. ${ }^{86}$

\subsection{Demokratie und Stochokratie}

Ratsmitglieder und die meisten Ämter wurden ausgelost, denn, wie Aristoteles erklärt: „(...) es ist demokratisch, die Ämter durch das Los zu verteilen, sie aber durch Wahl zu bestimmen ist eben oligarchisch“.87 Dem stimmte auch Charles de Montesquieu $1748 \mathrm{zu}:^{88}$

Die Abstimmung durch das Los entspricht dem Wesen der Demokratie, die durch Wahl dem der Aristokratie. Das Los ist eine Art der Wahl, die niemanden kränkt und jedem Bürger eine angemessene Hoffnung lässt, dem Vaterland dienen zu können.

Aus heutiger Sicht wird das Losverfahren oft kritisiert. So könne das Volk nicht seine „Vertreter“ wählen und habe somit keine Kontrolle über Amtsinhaber und Ratsmitglieder; die gelosten Vertreter könnten inkompetent sein; das Volk könne nicht wie in modernen Demokratien durch Wahl politische Programme sanktionieren. Solche Kritikpunkte spiegeln ein Unverständnis gegenüber der athenischen Demokratie wider: Annuität und das Verbot mehrjähriger Amtsperioden bedeuten, dass alle Bürger selbst, wie Aristoteles beschreibt, für gewisse Zeit an der Macht sind und niemand eine vorherrschende Machtstellung einnehmen kann. ${ }^{89}$ Auch geht es nicht um politische Programme: So war in Athen jede Form der Parteienbildung suspekt und wurde aktiv unterbunden (nach der Oligarchie 411 sollten Regeln zur Sitzordnung jede Art von Gruppenbildung in der boulé verhindern ${ }^{90}$ ). Durch die Kollegialität in allen Ämtern wird der Einfluss des einzelnen beschränkt. Gegen den Vorwurf der Inkompetenz gibt es mehrere Argumente: Nicht nur gab es ja die dokimasia vor Amtsantritt ( $v$. supra), sondern viele Bürger hatten zudem Erfahrung in Selbstverwaltung und in Politik sammeln können: in den Demen, Phratrien, im Rat, den dikastēria, etc.

86. Meiggs-Lewis no. 90

87. Pol. 4, 1294b5-10.

88. Vom Geist der Gesetze I 2,1 (1748), Tübingen 1992.

89. Zum Strategenamt, v. infra.

90. Philochorus FrGrHist 328 F 140; WelweI, griech. Polis, 239. 
Im Zusammenhang mit den Ratsmitgliedern, die alljährlich aus der Bürgerschaft für ein Jahr ausgelost wurden, ${ }^{91}$ muss man im Übrigen Athens demographische Lage bedenken. Athen hatte zwar rund 250.000 Einwohner, inklusive Sklaven und Metöken, ${ }^{92}$ aber davon schätzungsweise nur 30.000 männliche Bürger, die politisch aktiv werden durften; laut Aristoteles wurden alleine 20.000 Männer jedes Jahr als Amtsträger, Richter und bouleutes von den Tributleistungen der „Bündnispartner“ ernährt. ${ }^{93}$ Als athenischer Bürger war es also schwer, nicht in der Politik aktiv zu sein. Für die boulé brauchte man jedes Jahr 500 Männer, die mindestens 30 Jahre alt waren: ${ }^{94}$ Schon im Verlauf von 20 Jahren nach Kleisthenes' Reformen wären insgesamt 10.000 Bürger in der boulé gewesen! Eine zweite Amtszeit war vermutlich eine Notwendigkeit geworden. Wenn man dazu noch die 6.000 Richter, die zahllosen sonstigen Amtsträger und die Selbstverwaltung der Demen berücksichtigt, wird deutlich, dass auch in der Volksversammlung viele Teilnehmer eine gewisse politische Erfahrung hatten und keineswegs weniger kompetent gewesen sein müssen als heutige Amtsträger; außerdem waren viele in die zahlreichen Konflikte Athens im 5. Jh. involviert und hatten als Soldaten, Ruderer und Händler Auslandserfahrung. Dennoch kommen zwei Fragen auf: Kam es zu Willkürentscheidungen des dēmos - in der Volksversammlung oder in den Gerichten - und konnten Demagogen den dèmos manipulieren?

\subsection{Demagogie oder Willkür des dēmos}

Es war dem Namen nach eine Demokratie, in Wirklichkeit eine Herrschaft des Ersten Mannes. ${ }^{95}$

So beurteilt Thukydides seinen Zeitgenossen Perikles. Auch wenn der Begriff Demagoge (wörtlich „Volksführer") in der Antike primär positiv belegt ist, so stellt sich doch die Frage, nach der Manipulation des dēmos. Konnte die Volksversammlung wirklich von einigen wenigen Individuen beherrscht werden und/oderinwie fern konnten Demagogen sogar notwendig sein für den Prozess der politischen Meinungsbildung? ${ }^{96}$

91. Eine zweite Amtszeit war nur mit Unterbrechung möglich.

92. Metoikos - ein in Athen ansässiger „Fremder”. Metöken waren zwar unter Kleisthenes im Rahmen der Demenreform (508-507) athenische Bürger geworden, doch galt seit Perikles (451/450) als Voraussetzung für die Gewährung des Bürgerrechts, dass beide Elternteile athenische Bürger sein mussten (Ath. Pol. 26,4; Plut. Perikles 37, 3), wodurch Integration erschwert wurde.

93. Ath. Pol. 24,3.

94. Cf. Ruschenbusch, Soziale Zusammensetzung, insbes. S. 177: Ruschenbusch schätzt für das Jahr 330, dass der Jahrgang der Dreißigjährigen im Jahr 330 sogar aus nur 480 Bürger bestand, so dass einige Demen Schwierigkeiten hatten, ihre Quote zu erfüllen.

95. Thuk. 2, 65, 8-11.

96. Cf. Stahl, Klassische Zeit, 108-113. 
Schon Moses Finley hat deutlich gezeigt, dass auch ein Perikles sich den Entscheidungen der Volksversammlung in einem ständigen Wettkampf um Argumente und um die Gunst des Volkes beugen musste:

Nicht einmal Perikles besaß solche Macht. Als sein Einfluss auf dem Höhepunkt stand, konnte er dauerhaft auf Billigung seiner politischen Unternehmungen hoffen, die sich in der Abstimmung des Volkes in der Ekklesia äußern musste, doch wurden seine Gesetzesvorschläge Woche für Woche eben dieser Versammlung vorgelegt (...). Letztendlich lag die Entscheidung bei der Volksversammlung, nicht bei ihm oder irgendeinem anderen führenden Politiker (...). ${ }^{97}$

Auch schon Perikles - zitiert nach Thukydides - betont die Bedeutung von Reden und damit die Rolle der Redner und Demagogen in der politischen Meinungsbildung: das Volk muss die Argumente abwägen können:

Denn wir sehen nicht im Wort eine Gefahr fürs Tun, wohl aber darin, sich nicht durch Reden zuerst zu belehren, ehe man zur nötigen Tat schreitet. ${ }^{98}$

Aus der Erfahrung des 6. Jh. war es ja das Ziel seit Kleisthenes, jede Form von Parteienbildung zu vermeiden. Die „Vermischung“ der Bürgerschaft durch die Phylenreform sollte ja auch verhindern, dass Adelige persönliche Gefolgschaften mobilisieren konnten. Es gab somit keine genau definierte politische Führung. Die Volksversammlung wurde zum Schauplatz, an dem verschiedene politische Meinungen ausgetragen wurden. Überzeugung war eine Frage der Argumente, aber auch der Eloquenz und des Charismas des jeweiligen Redners. Perikles konnte als einer der zehn strategoi (Feldherrn) wiedergewählt werden, doch das heißt nicht, dass das Amt des Strategen eine Schwachstelle der Demokratie war. Athenische Feldherren mussten in der ersten Reihe mitkämpfen und die Konsequenzen tragen (v. infra).

Andererseits wurde die athenische Demokratie auch als Willkürherrschaft der Masse aufgefasst. Für Plutarch war der dèmos ein "bösartiges Tier",99 während Zeitgenossen gerade in Athens Politik mit seinen Bundesgenossen die uneingeschränkte Macht (tyrannis) des dèmos erkannten. ${ }^{100}$ Es gibt zwei Ereignisse, welche traditionell als Exempel für die Willkürherrschaft des dèmos herangezogen werden:

97. Finley, Athenian Demagogues; ID., Antike und moderne Demokratie. Siehe auch Will, Perikles, 7ff für den Mythos „Perikles“. Im Jahr 430 wurde Perikles selbst wegen Unterschlagung angeklagt (Plut. Perikles 32) und später vom Amt des Strategen enthoben und zu einer Geldstrafe von 15-50 Talenten verurteilt (ibid. 33,3ff - 34; Thuk. 2,65,3)

98. Thuk. 2, 40, 2

99. Plut. Dem. 26, 6-7.

100. Z.B. Aristoph. Equ. 1111-1114; Thuk. 3, 37, 2. 
Dazu gehört der Arginusen-Prozess. ${ }^{101}$ Nach dem Verlust von 25 Schiffen im Sommer 406 werden die strategoi angeklagt, sie hätten die Bergung der Überlebenden vernachlässigt, doch konnten sie die Volksversammlung von ihrer Unschuld überzeugen. Daraufhin wird die boulégebeten, ein probouleuma („Vorbeschluss“) für die nächste Sitzung auszuarbeiten. Nachdem im Rat gegen die Strategen durch einen gewissen Theramenes Stimmung gemacht wurde, entsteht ein probouleuma, das bei Schuldspruch allen strategoi gemeinsam die Todesstrafe verheißt. Als dieses probouleuma von Kallixenos in der nächsten Volksversammlung vorgetragen wurde, wonach es eine offene Abstimmung ohne Beratung geben sollte, beantragt ein gewisser Euryptolemos ein Paranomie-Verfahren wegen Gesetzwidrigkeit gegen Kallixenos, weil es für jeden Strategen ein getrenntes Verfahren wegen Amtsverletzung geben müsse und die Verteidigung der Strategen sowie eine geheime Abstimmung gewährleistet sein müssten. Es kommt zu Tumult in der Volksversammlung. Die eingeschüchterten Prytanen, die den Vorsitz in der ekklesia führten, willigten schließlich ein, entgegen dem gesetzmäßig vorgesehenen Verfahren die Abstimmung vorzunehmen. Schließlich findet das probouleuma des Rats die Mehrheit und die strategoi werden hingerichtet Der Arginusen-Prozess zeigt, dass vorhandene Verfahrensnormen vorhanden waren, um Machtmissbrauch zu verhindern, doch diese Kontrollmechanismen versagten in diesem Extremfall: Die Ausnahme bestätigt die Regel?

Der zweite Fall betrifft die dikastēria - die Gerichtshöfe. Die 6.000 Richter wurden jeden Tag auf die jeweiligen Prozesse per Los verteilt, wozu man Losmaschinen erfand, um den Prozess zu vereinfachen. ${ }^{102}$ Die Auslosung sollte ebenso wie die große Anzahl von mindestens 101 „Schöffen“ Korruption und Befangenheit verhindern. Um Gruppenzwang zu vermeiden, wurde (spätestens im 4. Jh.) die geheime Abstimmung durch Stimmsteine eingeführt. ${ }^{103}$ Die Anzahl von 6.000 Richtern machte eine Beteiligung der unteren Gesellschaftsschichten erforderlich. Wie in jedem Rechtssystem, gibt es jedoch auch in Athen Justizirrtümer. Ein Prozess steht dafür exemplarisch: das gegen den Philosophen Sokrates (469-399) wegen seiner Lehren und der Leugnung der Götter verhängte Todesurteil. ${ }^{104}$

Hierauf habt ihr, Athener, Sokrates, den Sophisten, hingerichtet, weil er offensichtlich den Kritias unterrichtet hat, einen der 30 Tyrannen, die die Demokratie zerstört hatten. ${ }^{105}$

Wie kommt es, dass ein Gerichtshof aus 501 Geschworenen einen Philosophen zum Tode verurteilt hat, der lediglich sein Recht auf freie

101. Xen. Hell. 1, 7, 7-16; Diod. 13, 101-103; STAHL, Klassische Zeit, 101-6.

102. Detaillierte Beschreibung: Ath. Pol. 63-69. Ath.Pol. 24, 3.

103. Ath.Pol. 68, 2-69, 1.

104. Plato, Apologie.

105. Aischin. 1, 173. 
Rede ausübt? Ein Paradox? ${ }^{106}$ Sokrates hat Jahrzehnte lang unbehelligt seine Philosophie verbreiten können. Der Anklagepunkt, er habe durch seine Lehren - wie im Falle des Kritias deutlich würde - die Jugend verdorben, wie auch das Urteil erklären sich aus der prekären Situation des Jahres 399: Nur wenige Jahre nach dem Ende des peloponnesischen Krieges und dem Ende der 30 Tyrannen (404-403) geht es darum, die erneuerte Demokratie zu verteidigen und zu stabilisieren. Alles in allem gab es insbesondere im 4. Jh. keine Willkürherrschaft, sondern, wie Karen Piepenbrink ${ }^{107}$ gezeigt hat, festgelegte Normen, die den Ablauf von Gesetzgebung und Gerichtsprozessen genau regelten.

\subsection{Ostrakismos - „fail-safe mechanism“ der jungen Demokratie?}

Zumindest für das 5. Jh. ergeben die Quellen den Eindruck, die Politik sei von großen Männern beherrscht worden, wie Themistokles, Kimon, Ephialtes und Perikles, so dass es leicht zur „Herrschaft des Ersten Mannes“ hätte kommen können. In diesem Kontext war der Ostrakismos („Scherbengericht“), wodurch ein prominenter Bürger für 10 Jahre in Exil geschickt werden konnte, ein fester Bestandteil des demokratischen Systems. ${ }^{108}$ Laut Ath. Pol. $(22,1)$ soll dieses Verfahren schon von Kleisthenes eingeführt worden sein, vermutlich mit der Absicht, die Herrschaft des dēmos gegen die Übernahme durch einen Tyrannen zu sichern; erstmals ist der Ostrakismos 487 belegt, als Hipparchos, ein Verwandter des Tyrannen Peisistratos, verbannt wurde.

(...) und als das Volk endlich an Mut gewann, wandten sie zum ersten Mal das Gesetz über das Scherbengericht an, das wegen des Misstrauens gegen die Inhaber der Machtstellungen erlassen worden war, weil sich ja Peisistratos als Volksführer und Stratege zum Tyrannen aufgeworfen hatte. Als erster wurde dann auch einer seiner Verwandten durch das Scherbengericht verbannt (...). ${ }^{109}$

Ein derartiger Prozess verlief in festgelegten Schritten, ohne dass ein athenischer Bürger einen bestimmten Mitbürger anklagen musste, wie es sonst üblich war: In der 6 . Prytanie jeden Jahres hatte die Volksversammlung zu entscheiden, ob ein Ostrakismos stattfinden sollte. ${ }^{110}$ Falls ja, wurde dieser zwei Monate später durchgeführt. Dieser Zeitraum verhinderte spontane Reaktionen aus „Volkszorn“ und ermöglichte Diskussion und politische Agitation bis zur Abstimmung. Im Gegensatz zum normalen Gerichtsprozess

106. So z.B. Stone, Trial, 197; Hansen, Trial; Ober, Living Freely. Überblick bei Samons, What's Wrong, 11f. Zur möglichen Einseitigkeit unserer Quellen, insbesondere Plato und Xenophon, die Sokrates verteidigen, siehe Morrison, Alleged; Waterfield, Why Socrates Died; Stahl, Klassische Zeit, 114-120.

107. Piepenbrink, Ordnungskonzeptionen.

108. Siehe Ath.Pol 24; für Ostrakismos und seine Quellen, siehe Beiträge von BRENNE, SCHEIDEL und Siewert in Siewert, Ostrakismos-Testimonien.

109. Ath. Pol. 22, 3-4.

110. Ath. Pol. 43, 5. 
in Athen gab es beim Ostrakismos weder Anklage noch die Möglichkeit der Verteidigung. Es traf auch nur eine Person, nämlich diejenige, deren Name am häufigsten auf den Scherben vorkam. Die auf 10 Jahre befristete Verbannung ohne Verlust von Bürgerrecht und Besitz war zudem extrem milde im Vergleich zu den Strafen auf „Hochverrat", die die Gerichtshöfe verhängen konnten, wie atimia $^{111}$, dauerhaftes Exil bzw. Todesstrafe. ${ }^{112}$

Auch wenn ursprünglich die Angst vor einer erneuten Tyrannis Auslöser für seine Entstehung war, so wurde der Ostrakismos Teil der politischen Auseinandersetzung, wie bei der Ostrakisierung Kimons 461, die richtungsweisend für die weitere politische und demokratische Entwicklung Athens war: in diesem Fall wurde die pro-spartanische Politik des oligarchisch gesinnten Kimon zugunsten der Politik des Perikles vom Volk abgelehnt:

(...) [die Athener kehrten] im Zorn [aus Sparta] zurück und empörten sich offen gegen die Freunde der Spartaner. Sie griffen einen geringfügigen Vorwand auf, um Kimon durch das Scherbengericht auf zehn Jahre zu verbannen, denn dies war der festgesetzte Zeitraum für alle auf diese Weise Verbannten. ${ }^{13}$

Es wäre vereinfachend, den Ostrakismos lediglich auf inner-aristokratische Rivalitäten zurückzuführen; es geht hier unter anderem auch um politische Entscheidungen des dèmos, der zwischen sehr unterschiedlichen Programmen zu wählen hatte. Zudem demonstrierte der dèmos in diesem alljährlichen Ritual seine Macht über die Mächtigen, was für Josiah Ober eine jährliche Neuinszenierung jenes revolutionären Moments darstellt, als die Tyrannis abgewendet wurde. ${ }^{114}$

Doch die Gesellschaft wandelte sich und der Ostrakismos verlor durch Missbrauch zunehmend seine Bedeutung. Der letzte belegte Osktrakismos fand 414 statt, als Hyperbolos einen seiner Rivalen ostrakieren wollte: Nikias und Alkiabiades erreichten aber durch politische Agitation, dass Hyperbolos selbst verbannt wurde! ${ }^{115}$ Anschließend wurde ein neues Verfahren eingeführt: graphe paranomon „Klage gegen gesetzwidrige (Gesetze)“. Es handelt sich hier um ein Normenkontrollverfahren, wonach Beschlüsse der Volksversammlung (psēphismata) nicht im Widerspruch zu geltendem Recht stehen sollen; insbesondere auch auf die Einhaltung der Verfahrensrichtlinien wird dabei wertgelegt. Im Gegensatz zum Ostrakismos musste ein einzelner Bürger den Urheber eines vermeintlich „verfassungswidrigen“ Gesetzes innerhalb eines Jahres anklagen, welcher dann persönlich zur Verantwortung gezogen wurde, unter Umständen mit wesentlich härteren Strafen als beim Ostrakismos. Richter waren die Volksgerichte. Somit konnte ein Schauprozess entstehen,

111. D.h. Verlust der Bürgerrechte.

112. Cf. z.B. Hansen, Age of Demosthenes.

113. Plut. Kimon 17, 3.

114. OBER, I Besieged.

115. Plut. Aristides 7; Nikias 11; Alkibiades 13. 
womit man seinen politischen Gegner schädigen oder gar beseitigen konnte, wobei der Anlass im 4. Jh. oft eher unbedeutende Gesetze waren, z.B. Ehrendekrete, wie im Fall von Demosthenes' (384-322) "Rede über die Krone" (330). ${ }^{116}$

\subsection{Politische Reflexion im Theater}

Komödien und Tragödien spielten eine wichtige Rolle im demokratischen Athen. ${ }^{117}$ Alljährlich rangen Dramatiker auf den Dionysien um den ersten Preis und waren somit Teil einer politischen Reflexion, welche die ganze Bürgerschaft einbezog. Die Komödien des Aristophanes, zum Beispiel, hatten meist aktuelle Anlässe, die in spielerischem Ernst umgesetzt wurden. Lysistrata ist beispielsweise ein "Anti-Kriegs“-Stück (411) und auch Aristophanes" Ritter wurde 424 inmitten des Peloponnesischen Krieges aufgeführt und war gegen den „Kriegstreiber“ Kleon gerichtet. In der folgenden Passage werden die "niederen“ Qualitäten beschrieben, die ein Staatsmann wie Kleon braucht, um das Volk zu manipulieren:

Sklave: Qualifizierst du dich für Staatsgeschäfte?

Wursthändler: Schon recht! Allein ich habe nichts gelernt; ein bisschen lesen, ja, doch schlecht genug!

Sklave: Das bisschen eben könnt' am End' dir schaden. Regieren ist kein Ding für Leute von Charakter und Erziehung! Niederträchtig, unwissend muss man sein! Drum folge du dem Ruf, den dir der Götter Spruch verkündet. (...)

Wursthändler: Gut! Mich meint das Orakel! - Aber wie, das wundert mich, soll ich das Volk regieren?

Sklave: Spottleicht! Du machst es grade wie bisher. Du hackst und rührst den Plunder durcheinander, hofierst dem Volk und streichst ihm süße Wörtchen wie ein Ragout ums Maul.

Du hast ja, was ein Demagog nur immer braucht: die schönste Brüllstimme, bist ein Lump von Haus aus, Krämer, kurzum, ein ganzer Staatsmann! - Die Orakel sind, selbst das pythische, für dich! Drum trinke bekränzt! Ein Glas dem Genius der Dummheit!118

In Aristophanes“ Ekklēsiázousai („Weiberversammlung“, 392 v. Chr.) wird Athens Gesellschaftsordnung auf den Kopf gestellt, indem als Männer verkleidete Frauen, unter der Führung der Heldin Praxagora, dafür sorgen, dass die Volksversammlung ihnen die Macht gibt, da sie besser regieren würden: ${ }^{19}$

116. Für graphé paranomon, cf. Hansen, Age of Demosthenes.

117. STAHL, Klassische Zeit, Kapitel 6, 121-162.

118. Aristoph. Equ. 205-212.

119. Zu Ekklēsiázousai, cf. McGlew, Citizens 191ff.; Ober, Dissent. Siehe auch Plato, rep. 5.454e-455e zur Gleichheit von Männern und Frauen, welche die selbe Ausbildung erhalten sollen und damit auch Zugang zu allen gesellschaftlichen Positionen, z.B. 5.455d: “(...) also gibt es gar kein Geschäft von allen, durch die der Staat besteht, welches dem Weibe als Weib oder dem Manne als Mann angehörte, sondern 
Praxagora: (...) Du aber, Volk, du bist an allem schuld! Denn aus dem Staatsschatz zieht der Bürger Sold, und jeder sucht allein Gewinn für sich! (...) Folgt meinem Rat, dann blüht euch wieder Glück! Den Weibern, rat' ich, müssen wir den Staat ganz überlassen! Führen Sie zu Hause doch auch die Wirtschaft als Verwalterinnen (...) Dass sie in allem besser sind als wir, will ich beweisen (...). O Athen, wie wärst du wohlgeborgen, hieltest du's wie sie und fragtest nicht nach Neuerung! (...). ${ }^{120}$

Der dēmos, in seiner grenzenlosen Allmacht, stimmt zu! Ein geradezu „sozialistisches" Regime wird eingeführt: Privateigentum wird abgeschafft, Besitz und Frauen gehören allen und alle Bürger sollen auf öffentliche Kosten speisen. Hier werden Werte der Demokratie und der Bürgergemeinde, wie das Gleichheitsprinzip, zum Extrem geführt. Dies ist nicht nur eine Reflexion utopischer Gesellschaften - bisweilen als Satire - (wie z.B. Platons Republik, ca. $360^{121}$ ), die zeigt, dass solch radikale Utopien keineswegs alle Probleme beheben, stattdessen aber auch neue Probleme kreieren, sondern es geht auch um die Auslotung demokratischer Werte und der menschlichen Natur. Athen hat sich seit dem Peloponnesischen Krieg und den 30 Tyrannen gewandelt. Erkennbar ist die zunehmende Individualisierung der Gesellschaft: für Politik, Philosophie, Religion wie auch jeden Einzelnen steht die Polis nicht mehr im Mittelpunkt. Damit sind die Ekklēsiázousai auch eine Satire auf den habgierigen Athener, für den die eigenen Interessen wichtiger sind als das Gemeinwohl und der nur wegen der „Diäten“ zur Volksversammlung kommt. ${ }^{122}$ Ebenso wie in Aristophanes' Plutus "Reichtum“ (388) geht es um eine gesellschaftliche Utopie, in der die Protagonisten gegen die Armut kämpfen, doch erscheinen Wohlstand und Gerechtigkeit unvereinbar. ${ }^{123}$ Aristophanes offenbart sich in seinen Komödien als aktiver Protagonist der athenischen Demokratie. ${ }^{124}$

\section{EPILOG: SOUVERÄNITÄT DES DĒMOS}

In Perikles“ „Totenrede“ spiegelt sich besonders eindrücklich das demokratische Selbstverständnis seiner Zeit wider:

Die Verfassung, nach der wir leben, vergleicht sich mit keiner der fremden; viel eher sind wir für sonst jemand ein Vorbild als Nachahmer anderer. Mit Namen

die natürlichen Anlagen sind auf ähnliche Weise in beiden verteilt, und an allen Geschäften kann das Weib teilnehmen ihrer Natur nach wie der Mann an allen (...)”.

120. Aristoph. Eccl. 194-222.

121. Siehe auch Phaleas von Chalkedon (5. Jh.), der Gleichheit bei Landbesitz und Bildung fordert, um gesellschaftliche Spannung zu vermeiden (Aristot. Pol. 1266b30-40); im Sinne einer erfolgreichen Umsetzung des Gleichheitsprinzips müssten nach Aristoteles aber alle Formen von Besitz festgelegt werden (ibd. 1267b10-14).

122. Aristoph. Eccl. 289-315 für den triobolus. Zu Ekklēsiázousai cf. Rothwell, Politics, S. 11.

123. Cf. MCGLEw, Citizens, 175-6.

124. Ober, Dissent 152f; MCGlew, Citizen $192 \mathrm{f}$. 
heißt sie, weil der Staat nicht auf wenige Bürger, sondern auf eine größere Zahl gestellt ist, demokratia. Nach dem Gesetz haben in den Streitigkeiten der Bürger alle ihr gleiches Teil, der Geltung nach aber hat im öffentlichen Wesen den Vorzug, wer sich irgendwie Ansehen erworben hat, nicht nach irgendeiner Zugehörigkeit sondern nach seinem Verdienst. (...) Reichtum dient bei uns der wirksamen Tat, nicht dem prahlenden Wort, und Armut ist einzugestehen keinem schimpflich, ihr nicht tätig zu entgehen schimpflicher. (...) nur wir entscheiden in den Staatsgeschäften selber oder denken sie doch richtig durch. ${ }^{125}$

Zusammenfassend sage ich, dass insgesamt unsere Stadt die Schule von Hellas ist. $^{126}$

Heute ziehen wir ebenfalls eine eher positive Bilanz für die athenische Demokratie. ${ }^{127}$ Athen war nicht der Allmacht des „Pöbels“ ausgesetzt und der dèmos war auch kein Tyrann: Justizirrtümer und Gräueltaten gegen Strategen oder Bundesgenossen gab es nicht nur in Athens Demokratie, sondern auch in Oligarchien und Mischverfassungen wie im benachbarten Sparta. Die Politik der athenischen Demokratie erscheint größtenteils effizienter, zielstrebiger und erfolgreicher als die seiner oligarchischen Nachbarn: so wurde selbst Theben erst im 4. Jh. zur "Großmacht", als es demokratisch regiert wurde. ${ }^{128}$

Wie haben die Athener trotz - oder geradezu durch - Basisdemokratie diese erstaunliche politische und verfassungsmäßige Leistung vollbracht? Die Demokratie hat im Laufe des 5. Jahrhunderts eine Eigendynamik entwickelt, die dazu geführt hat, dass die Macht des dēmos immer weiter gefestigt wurde. Der dèmos entwickelte ein Selbstbewusstsein und setzte sich gegen übermächtige Adelige durch, indem der Einfluss einzelner Politiker und vorstaatlicher Strukturen (Phratrien, genee, etc.) eingeschränkt wurde. Der dynamische Charakter der Demokratie erklärt zudem die enorme Anpassungsfähigkeit an Veränderungen im Gegensatz zu oligarchischen Systemen, deren Mitglieder häufig den status quo erhalten wollen. Grundprinzipien in Athen waren neben Redefreiheit, Rechtsgleichheit, Annuität und Popularklage vor allem die "Vermischung" der Bevölkerung durch die Neuordnung der Phylen, die Rotation, ${ }^{129}$ die großen kollektiven Gremien, die Auslosung aus allen Zensusklassen, sowie die Einführung von Diäten, geheimen Abstimmungen, Ostrakismos und graphe paranomon. Diese vielfältigen Maßnahmen dienten insgesamt dazu, den Einfluss von Individuen und Oligarchen ebenso einzuschränken wie Parteienbildung und Korruption. Darin erkennt man

125. 431/30 v. Chr.: Perikles` Rede zitiert nach Thuk. 2, 37-38, 40.

126. Thuk. 2, 41, 1 .

127. Cf. z.B. Murray, Animals; OBer, Athenian revolution, 3-12. Siehe auch den großen epigraphischen Befund, der - im Gegensatz zu den philosophischen Schriften eines Aristoteles oder Platons - das Funktionieren der athenischen Demokratie belegt: cf. z.B. Hedrick, Writing; RAAflaub, Introduction, 6-10.

128. BuCKLer, Theban; Hansen, Bryn Mawr Classical Review 2006.01.32.

129. Aristot. Pol. 1317b2-7. 
den konsequenten Versuch des dèmos, Athens demokratisches System ständig zu verbessern und Machtmissbrauch zu unterbinden. Wichtig sind in diesem Zusammenhang die politische Erfahrung und der Lernprozess aller athenischen Bürger, der auch einen gewissen Grad an Schriftlichkeit und Bildung (siehe z.B. die Bedeutung der Sophisten, wörtlich "Weisheitsbringer") miteinschließt. Gerade nach dem „Putsch“ der Oligarchen (411-410) und der Herrschaft der 30 Tyrannen $^{130}$ (404-403) kam es zur weiteren Vollendung der demokratischen Institutionen und der Souveränität des Volkes: ${ }^{131}$

Das Volk hat sich nämlich selbst zum Souverän gemacht, alles verwaltet es durch Abstimmungen und Gerichtshöfe, in denen das Volk beherrschend ist. Auch die Entscheidungen des Rates sind auf das Volk übergegangen, und das scheint man so richtig zu handhaben; denn eine kleine Gruppe lässt sich durch Vorteile und Vergünstigen leichter bestechen als die große Masse. ${ }^{132}$

Wie in modernen Demokratien hatte auch der athenische Bürger individuelle Rechte, die ihn gegen den Staat und seine Mitbürger schützten. Ihm war damit die Möglichkeit gegeben selbstbestimmt zu leben, und gegebenenfalls auch gegen Beamte Klage zu führen. ${ }^{133}$ Schon für Aischines $(1,4-5)$ war dieser rechtliche Schutz ein "Gütezeichen“ der Demokratie. ${ }^{134}$

Politische Rechte hatten jedoch nur die männlichen Bürger, nicht die Frauen, Kinder, Sklaven und Metöken. Wie wir gesehen haben, war die politische Emanzipation eine lange Entwicklung, in der Beschränkungen und Schranken immer weiter abgebaut wurden: ${ }^{135}$ Ursprünglich waren es nur die Adeligen im Areopag, dann die Hopliten - wie in Solons "Timokratie“ - und schließlich die untere „Zensusklasse“, die Theten, welche politische Rechte erhielten, da sie für Athen in den Krieg zogen. Für das antike Verständnis erscheint es schon revolutionär, dass Menschen (von arm bis reich) unabhängig von ihrem finanziellen und sozialen Status nicht nur wählen durften, sondern auch Exekutivmacht erhielten. Auch wenn es nur die Männer waren, denen diese Rechte zu Teil wurden, so war auf diese Art theoretisch jeder Haushalt in Athen politisch vertreten. Verheirate Frauen wurden anfangs sogar in unseren westlichen „Demokratien“ nur durch ihre Ehemänner vertreten. Stimmrecht für Frauen gab es meistens erst nach dem 1. Weltkrieg (z.B. Deutschland, 19.1.1919 und erst 1920 in allen Bundesstaaten der U.S.A.), nachdem Frauen in der Kriegszeit „ihren Mann gestanden“ hatten, während die Politik noch bis

130. Für die 30 Tyrannen, cf. z.B. NÉmEth, Kritias.

131. Cf. Eder, Athenische Demokratie; Hansen, Age of Demosthenes. Wohl schon ab 410 eine Schwächung der boulé, welche der Volksversammlung untergeordnet wird, cf. Welwei, griech. Polis, 239; IG $\mathrm{I}^{3} 105$.

132. Ath. Pol. 41, 2.

133. Cf. Hansen, Polis and City-State, 92-4.

134. Für die „common ground rules“, welche die Rechten und Pflichten eines athenischen Bürgers festlegten, cf. OBER, Civic education, 135-6.

135. Hornblower, in Dunn, 2; cf. Sallares 1991, 181. 
ins späte 20. Jh. überwiegend eine Männerdomäne blieb. In vielen Ländern waren Frauen sogar noch länger vom Wahlrecht ausgeschlossen (Schweiz 1971, Liechtenstein 1984), während „ethnische Randgruppen“ beispielsweise die Aborigines in Australien (bis 1967) ebenfalls lange von bürgerlichen und politischen Rechten ferngehalten wurden. Im Falle von Athen könnte man spekulieren, was ohne Alexander den Großen und das Ende der Demokratie (322) an weiterer Entwicklung möglich gewesen wäre: Hätten auch Frauen politische Rechte bekommen? Der Grundgedanke dazu war jedenfalls wie wir gesehen haben, schon von Platon und Aristophanes formuliert, und Frauen spielten auch eine wichtige soziale Rolle, beispielsweise als Priesterinnen. ${ }^{136}$

Selbst moderne Demokratien können sich kaum an der Souveränität des athenischen dèmos messen, in der Herrschende und Beherrschte praktisch identisch sind. Worauf ist die weitgehende verfassungsmäßige Stabilität und politische Harmonie in dieser von derart divergierenden Interessen geprägten und doch demokratisch regierten Bürgergemeinde entgegen allen Hindernissen zurückzuführen, insbesondere in der politisch, militärisch und wirtschaftlich prekären Lage des 5.-4. Jh.? ${ }^{37}$ Für Paul Cartledge liegt das Geheimnis von Athens Erfolg in seinen mannigfaltigen Gremien und Diskussionsforen, die einen kreativen Prozess der gesellschaftlichen und politischen Anpassung und Innovation ermöglichten. Dadurch, dass die Teilnehmer im politischen Prozess - in ekklesia und boulé - aus sehr unterschiedlichen sozialen Gruppen stammen und dementsprechend disparate (und manchmal unvereinbare) Anschauungspunkte vertreten, entstand eine sehr hohe Spannung, die aber nach Cartledge durch die Regeln der demokratischen Bürgergemeinde in einen progressiven und zugleich solidarisch herbeigeführten Konsens kanalisiert wurde. ${ }^{138}$

Für heutige demokratische Verhältnisse erscheint Athens "bottom-up approach" einer Gesellschaftsordnung, die von unten nach oben aufgebaut ist, bemerkenswert: Ist es nicht erstaunlich, dass man den Mut hatte, dem "einfachen Mann" nicht nur Stimmrecht, sondern auch Macht in Rat, Behörden und Gerichten zu geben, ihn zur aktiven Teilnahme nötigte? Der Mut zur Auslosung und zur Rotation erklärt sich unter anderem daraus, dass man an die Mehrheitsentscheidung des Kollektivs glaubte - ein Kollektiv, das seit Kleisthenes' Phylenreform und der „Vermischung" der Bevölkerung einen Querschnitt der Bevölkerung widerspiegeln sollte. Darin werden Unterschiede zur heutigen Mehrheitsbildung deutlich. In viel stärkerem Maße delegiert der Bürger heute im Zuge von Wahlen seine Interessenswahrnehmung an Parteien, die nach Mehrheitsverhältnissen dann quasi „im Volksauftrag herrschen“, während zugleich die Auffassung der Wahlverlierer für Jahre auf die Rolle der kritischen Opposition beschränkt ist. Dies kann heutzutage zu großen sozialen

136. Cf. Dillon, Girls.

137. OBER, Athenian Legacies, 2; HANSEN, Age of Demosthenes.

138. Cartledge, Kosmos, 12. 
Spannungen führen, wie in Nordirland, Südafrika, Irak oder Zimbabwe, welche dann durch eine Multi-Parteien Regierung bzw. „government of national unity" abgebaut werden sollen. Aber schon Montesquieu hat erkannt (v. supra), dass das Los eine Wahl ist, die niemand kränkt, vor allem wenn Regierung, Institutionen und Kommissionen ein Querschnitt aller Einwohner jenseits aller ethnischen, sozialen oder religiösen Unterschiede widerspiegeln würden.

Wie Ober gezeigt hat, ${ }^{139}$ gehört zum Funktionieren der athenischen Demokratie das wechselseitige Verhältnis zwischen Wissen und Tun, d.h. die aktive Beteiligung von "knowledgeable amateurs", die an dem tatkräftigen und permanenten Austausch von Wissen - in den demokratischen Institutionen, wie boulé und dikastēria - teilnehmen und Erfahrungswerte über das Funktionieren der Demokratie und der demokratischen Kultur sammeln. Bei der Rückkehr in die heimische Deme kann so zum Beispiel ein ehemaliges Ratsmitglied seine Demenangehörigen in der Demokratie unterweisen. ${ }^{140}$ Demokratie lernen in Athen heißt vor allem „learning by doing“.

Das Auslosen der Beamten, Richter und Ratsmitglieder bedeutet, dass durch Zufall sehr verschiedene Menschen mit sehr unterschiedlichem Hintergrund und Erfahrungen in einem Gremium zusammenarbeiten müssen. ${ }^{141}$ Anstatt die Inkompetenz eines Einzelnen anzuschwärzen, können sich auf diese Weise potentielle Synergieeffekte ergeben, wie schon Aristoteles aus der praktischen Erfahrung mit Athens Demokratie erkannt hat (infra). Möglicherweise finden sich in der heutigen Internet-Kultur (z.B. Wikipedia, Internetforen, etc.) vergleichbare Strukturen wieder. Dabei kann die große Zahl der Individuen mit ihrem jeweiligen Wissen insgesamt einen größeren Beitrag leisten als der einzelne Gebildete. ${ }^{142}$

(...) Dass aber das Entscheidende eher die Menge ausmacht als die wenigen Besten, (...) birgt vielleicht aber auch eine Wahrheit. Denn es ist möglich, dass nämlich die Vielen, von denen jeder durchaus nicht ein rechtschaffender Mann ist, wenn sie zusammengekommen sind, besser sind als jene Besten, nicht allerdings als einzelner, sondern als Gesamtheit genommen (...). Denn da sie viele sind, kann jeder über einen Teil der Tugend und der Einsicht verfügen, und wenn sie zusammenkommen, kann die Menge werden wie ein einziger Mensch, der viele Füße, Hände und viele Sinneswerkzeuge hat und so auch im Hinblick auf die Wesensart und die Denkweise. ${ }^{143}$

Und jeder Einzelne für sich ist sicherlich schlechter, wenn er mit dem besten Mann verglichen wird, aber ein Staat besteht aus vielen (...). Und deshalb beurteilt die Menge auch vieles besser als irgendein Einzelner. Zudem ist auch die Menge unbestechlicher als irgendein Einzelner (...). Aber das Urteil eines

139. Ober, Democracy Today, 34.

140. Ibd., 38 .

141. Ibd., 41

142. Siehe auch OBer, Political Dissent, 319-326.

143. Aristot pol. 3, 1281a40-1281b10. 
Einzelnen ist verdorben, wenn ein Einzelner vom Zorn oder einer sonstigen solchen Emotion übermannt wird; dort aber ist es schwer, dass alle Zugleich in Zorn geraten und Verfehlungen begehen. ${ }^{144}$

Fragen wir uns zum Abschluss, welche Aspekte aus Athens politischer Kultur uns zum Nachdenken über die gegenwärtige politische Realität Anregung geben können. Wo kann in unserer Gesellschaft die demokratische Kultur einer „basisnahen“ Mitbestimmung „des Volkes" hilfreich sein, beispielsweise auf lokaler Ebene oder bei Gremienarbeit? ${ }^{145}$ Demokratie nach athenischem Modell kann ein Modell sein für ein Organisationsumfeld, in dem Menschen als freie, gleiche und selbstsichere Personen agieren, wie z.B. Josiah Ober argumentiert hat: Mut zur demokratischen Diversität und zur Diversität des Wissens. ${ }^{146}$

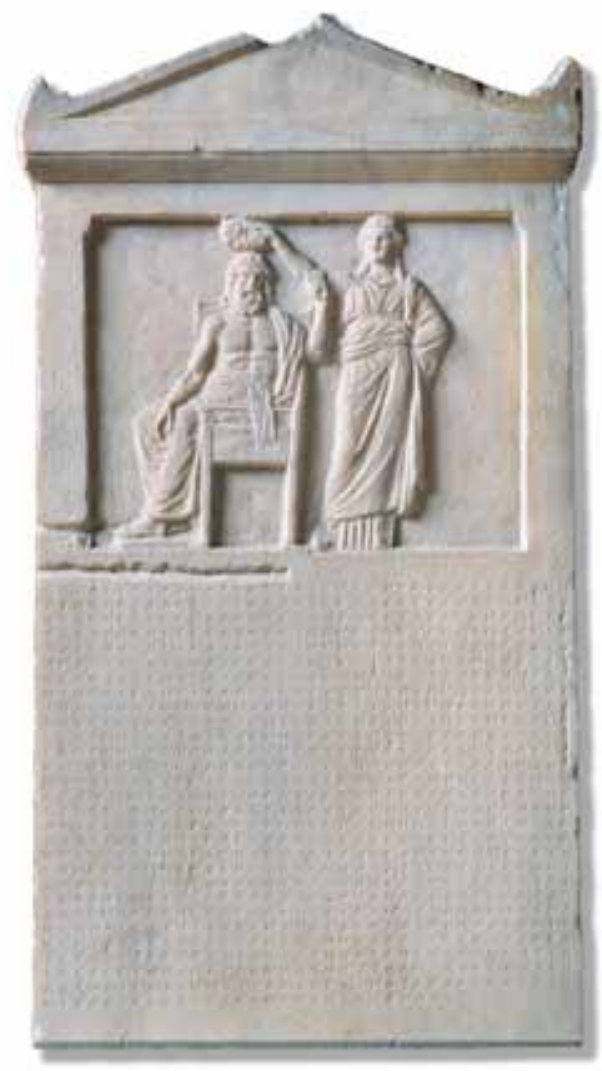

Abb. 1. Demokratia krönt den Demos: Gesetz gegen die Tyrannis, 337/6 v. Chr. (Photo: Craig Mauzy, Agora Excavations, The American School of Classical Studies)

144. Aristot pol. 3, 1286a24-31.

145. Sutherland, People's Parliament; Ober, Democracy Today, 32; Manville/Ober, Company.

146. Ober, Democracy Today, 34-35. 
Vor dem Hintergrund dieser Sicht auf die Demokratie Athens ergibt sich für unsere modernen repräsentativen „Demokratien“ bisweilen der Eindruck von Oligarchien, in denen Entscheidungen von privilegierten Minderheiten gefällt werden. ${ }^{147}$ Joseph Schumpeter schrieb: „Wer entscheidet, sind die Führer der politischen Parteien, nicht das Volk“. ${ }^{148}$ Grundlage ist das, was er "leadership democracy" oder "competitive elitism" nennt, wodurch Individuen gewählt werden, die den "Volkswillen“ ausführen sollen. ${ }^{149}$ Das Ergebnis ist eine „demokratische Elitenherrschaft", basierend auf einem „tiefen Misstrauen gegenüber der Mehrheit gemeiner Männer und Frauen" und dem "Vertrauen in die etablierten Eliten“. 150 Sind unsere modernen „Demokratien“ dann ein Synonym für Aristoteles" „Herrschaft der Besten“? Wenn man sich die gesellschaftliche Entwicklung Griechenlands vergegenwärtigt, muss man sich doch fragen, inwieweit sich in der heutigen Verfassung antike Elemente widerspiegeln. Vielleicht kann man moderne "Demokratien“ als Mischverfassungen auffassen, vergleichbar mit Sparta und vor allem mit der römischen Republik: das demokratische Element sind die Wahlen und vielleicht die Volksbeschlüsse (sofern letztere überhaupt verfassungsmäßig vorgesehen sind), das oligarchische Element spiegelt sich wider in Parlament, Ämtern und Funktionärselite, das monarchische wird verkörpert von je nach Verfassung - Präsident oder Premierminister, wobei es sogar in Rom zwei Konsuln gab, die nur ein Jahr amtieren und sich gegenseitig durch Veto blockieren konnten. Tatsächlich besteht heute die Sorge, dass die Bürger allenfalls noch als Wähler ihre demokratischen Grundrechte wahrnehmen, das Erlebnis individueller politischer Souveränität und das Erkennen der Chancen persönlicher politischer Einflussnahme dagegen in der heutigen demokratischen Alltagskultur zunehmend verloren gehen. So fühlen sich die Bürger in vielen westlichen Demokratien von Parteien und Parlamentsabgeordneten entfremdet oder gar entrechtet. Mitunter wird heute erneut die Auswahl von Abgeordneten nach dem Los gefordert, wie in Keith Sutherlands Buch „A People's Parliament“ oder für die Reform des britischen Oberhauses ${ }^{151}$ - für Times Literary Supplement „a sign that the public philosophy which has for so long governed our political arrangements has become outworn". ${ }^{152}$

147. Cf. Samons, What's Wrong (mit Rezension von M.H. Hansen, Bryn Mawr Classical Review 2006.01.32); OBER, Democracy today, 41.

148. SChumpeter, Capitalism.

149. Ibd., 150. Cf. Held, Models, 148 zu Schumpeter, Capitalism, 254-268: Nach Schumpeter sei der „Volkswille” ein soziales Konstrukt mit nur wenig rationaler Grundlage; Beschlüsse von nicht-demokratische Institutionen könnten für das Volk akzeptabler sein als demokratische Entscheidungen.

150. Cf. z.B. Bachrach, Theorie, 111.

151. Anthony Barnett/Peter Carty, The Athenian Option. Radical Reform for the House of Lords, Exeter 2008. Siehe auch Oliver Dowlen, The Political Potential of Sortition. A study of the random selection of citizens for public office, Exeter 2008.

152. TLS 14th November 2008 


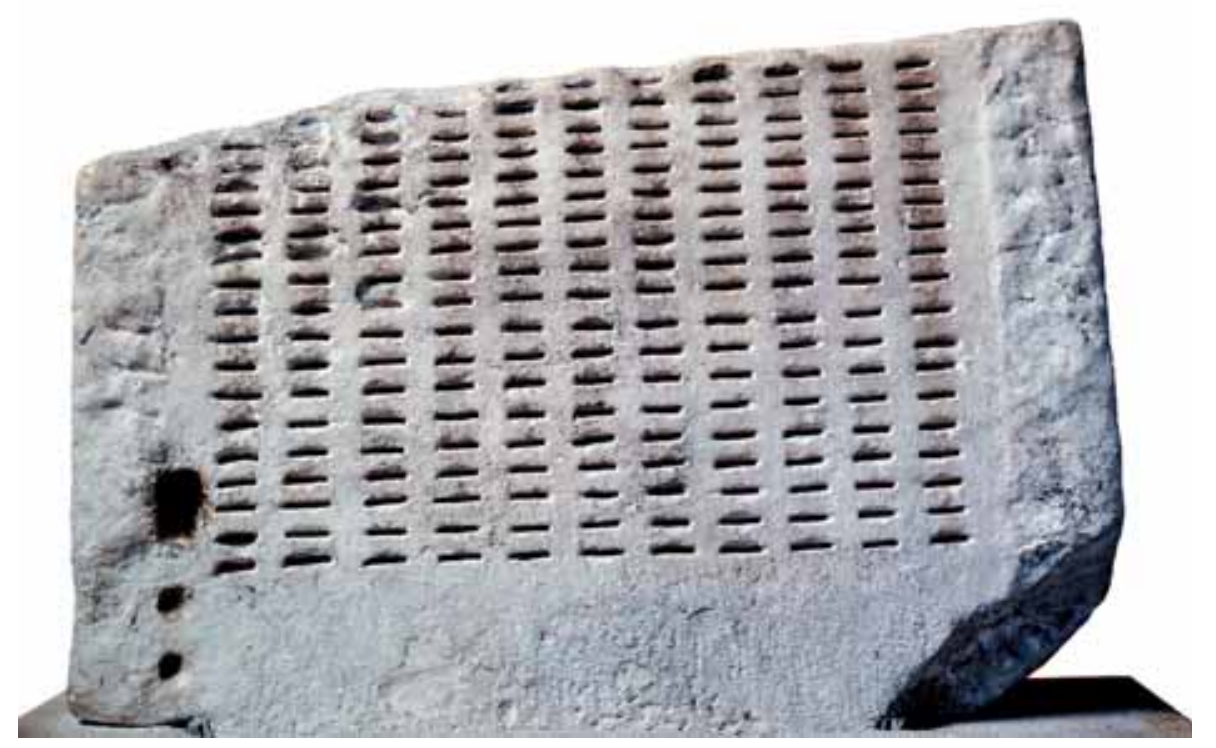

Abb. 2. Fragment eines Kleroterion (Losmaschine) mit in elf Spalten angeordneten Schlitzen, in welche Plättchen mit den Namen der zur Losung zur Verfügung stehenden Personen geschoben wurden. Gefunden 1935 im Metroon (Photo: Craig Mauzy, Agora Excavations, The American School of Classical Studies)

In Athen aber erlebte die Bürgergemeinde eine Wiedergeburt unter Solon und Kleisthenes, nicht nur durch die Entschärfung wirtschaftlich-sozialer Probleme, ${ }^{153}$ sondern durch ein Aufeinanderzugehen - Josiah Obers „Politics of going on together" ${ }^{\prime 154}$ - und vor allem durch die aktive Teilnahme der Bürger an allen Institutionen, also Demen, Phylen, Kulte, Rat, Gerichte und Volksversammlung: „Der athenische Bürger durfte (...) den Staat mit gutem Recht als den seinen betrachten“. 155

\section{BiBLIOGRAPHIE}

Anderson, Greg: The Athenian Experiment. Building an Imagined Political Community in Ancient Attica. 508-490 B.C., University of Michigan Press, Ann Arbor, 2003.

BACHRACh, Peter: Die Theorie demokratischer Elitenherrschaft, Europäische Verlags-Anstalt, Frankfurt, 1970.

Berve, Helmut: Die Tyrannis bei den Griechen, 2 Bde., Beck, München 1967. Bleicken, Jochen: Die athenische Demokratie, 4. Auflage, Schoeningh, Paderborn, 1995.

153. Walter, An der Polis teilhaben, 219.

154. ОвеR, Athenian Legacies.

155. So Walter, An der Polis teilhaben, 208. 
Buckler, John: The Theban Hegemony 371-362 B.C., Harvard University Press, Cambridge, Mass., 1980.

Cartledge, Paul: Introduction: Defining a Kosmos, In: Paul Cartledge / Paul Millett / Sitta von Reden, (Hgg.), Kosmos: Essays in Order, Conflict and Community in Classical Athens, Cambridge University Press, Cambridge, 1998, 1-12.

Develin, Robert / Kilmer, Martin: What Kleisthenes Did, Historia 46 (1997), 3-18.

Dillon, Matthew: Girls and Women in Classical Greek Religion, Routledge, London/New York, 2002.

Dover, Kenneth James: Aristophanes, Clouds, Clarendon Press, Oxford, 1968.

Dunn, John (Hg.): Democracy: the Unfinished Journey 508 BC to AD 1993, Oxford University Press, Oxford, 1992.

Eder, Walter: Die athenische Demokratie im 4. Jahrhundert v. Chr.: Krise oder Vollendung? In: WAlter Eder (Hg.), Die athenische Demokratie im 4. Jahrhundert v. Chr.: Vollendung oder Verfall einer Verfassungsform? Steiner, Stuttgart, 1995, 11-28.

Farrar, Cynthia: The Origins of Democratic Thinking: the invention of politics in classical Athens, Cambridge University Press, Cambridge, 1988.

Finley, Moses I.: Democracy Ancient and Modern, Chatto \& Windus, London, 1973 (= M.I. FInLEY, Antike und moderne Demokratie, mit einem Essay von Arnaldo Momigliano, Reclam, Stuttgart 1980).

Finley, Moses I.: Athenian Demagogues In: Rhodes, Athenian Democracy, 163-184.

Fitzjohn, Matthew: Equality in the colonies: concepts of equality in Sicily during the eighth to six centuries BC, World archaeology 39/2 (2007), 215228.

Frost, Frank J.: Aspects of Early Athenian Citizenship. In: Alan L. Boegehold / Adele C. Scafuro (Hgg.), Athenian Identity and Civic Ideology, John Hopkins University Press, Baltimore/London, 2002, 45-56.

Graham, Alexander John: Thucydides 7.13.2 and the Crews of Athenian Triremes, Transactions of the American Philological Association 122 (1992), 257-270.

Hansen, Mogens Herman: The Athenian Democracy in the Age of Demosthenes, Blackwell, Oxford, 1991.

Hansen, Mogens Herman: The Tradition of the Athenian Democracy A.D. 1750-1990, Greece and Rome 39 (1992), 14-30.

Hansen, Mogens Herman: Polis and City-State: an ancient concept and its modern equivalent, Munksgaard, Copenhagen, 1998 (Acts of the Copenhagen Polis Centre, 5).

Hansen, Mogens Herman: The Trial of Sokrates - from the Athenian point of view, Munksgaard, Copenhagen, 1995. 
Hansen, Mogens Herman: Misthos for Magistrates in Classical Athens, Symbolae Osloenses 54 (1979), 5-22.

Hansen, Mogens Herman: The Athenian Ecclesia, a collection of articles 1976-1983, Museum Tusculanum Press, Copenhagen, 1983.

Harris, E-M.: A New Solution to the Riddle of the Seisachtheia. In: Lynette G. Mitchell / Peter J. Rhodes (Hgg.), The Development of the Polis in Archaic Greece, Routledge, London, 1997, 103-112, Kapitel IX.

Hedrick, Charles W., JR.: Democracy and the Athenian Epigraphic Habit, Hesperia 68 (1999), 387-439.

Held, David: Models of Democracy, 3. Auflage, Polity Press, Cambridge/ Malden, MA., 2006 (1. Aufl. 1987).

Hornblower, Simon: Creation and Development of Democratic Institutions in Ancient Greece. In: DunN, Democracy, 1-16.

Jones, Nicholas F.: Rural Athens under the Democracy, University of Pennsylvania Press, Philadelphia, 2004.

LAmbert, S. D.: The Phratries of Attica, 2. überarbeitete Auflage, University of Michigan Press, Ann Arbor 1998.

Lavelle, Brian M.: The Sorrow and the Pity: A Prolegomenon to a History of Athens under the Peisistratids, c. 560-510 B.C., Steiner (Historia Einzelschriften 80), Stuttgart, 1993.

MacDowell, D. M.: Aristophanes and Athens, Clarendon Press, Oxford, 1995.

McGlew, James F.: Citizens on stage: comedy and political culture in the Athenian democracy, University of Michigan Press, Ann Arbor, 2002.

Manville, Brooke / Ober, Josiah: A Company of Citizens: What the World's First Democracy Teaches Leaders About Creating Great Organizations, Harvard Business School Press, Cambridge, MA., 2003.

Meier, Christian: The Greek Discovery of Politics, Cambridge, Mass., 1990 (= Die Entstehung des Politischen bei den Griechen, Suhrkamp Verlag, Frankfurt, 1983).

Morris, Ian / RaAflaub, Kurt A. (Hg.): Democracy 2500? Questions and Challenges, Kendall/Hunt, Dubuque, Iowa, 1998.

Morrison, D.: On the Alleged Historical Reliability of Plato's Apology, Archiv für Geschichte der Philosophie 82 (2000), 235-265.

Mossé, Claude: How a Political Myth Takes Shape: Solon, 'Founding Father' of the Athenian Democracy. In: P.J. Rhodes (Hg.): Athenian democracy, 242-259 (=Comment s'élabore un mythe politique: Solon, "père fondateur" de la démocratie athénienne“, Annales 34.3 (1979), 425-437).

Murray, Oswyn: Animals of the Polis, Times Literary Supplement (15 April 1994), S. 3.

Murray, Oswyn: Das frühe Griechenland, Deutscher Taschenbuch Verlag, München, ${ }^{3} 1986$. 
Németh, György: Kritias und die Dreißig Tyrannen. Untersuchungen zur Politik und Prosopographie der Führungselite in Athen 404/403 v. Chr., Steiner (HABES Bd. 43), Stuttgart, 2006.

Ober, Josian: Political Dissent in Democratic Athens: intellectual critics of popular rule, Princeton University Press, Princeston, NJ, 1998.

OBER, Josiah: The Athenian Revolution: essays on ancient Greek democracy and political theory, Princeton University Press, Princeton, NJ, 1996.

Ober, Josiah: The Athenian Revolution of 508/7 B.C.: Violence, Authority, and the Origins of Democracy, in: Robinson (Hg.), Ancient Greek Democracy, 95-112 (=in: OBER, Athenian Revolution, 32-52, chapter IV = In: RHODES, Athenian democracy, 260-286).

Ober, Josiah: Athenian Legacies: Essays on the Politics of Going On Together, Princeton University Press, Princeton, NJ, 2005.

Ober, Josiah: Classical Athenian Democracy and Democracy Today, in: OBer, Athenian Legacies, 27-42.

Ober, Josiah: The Athenian Debate over Civic education, in: Ober, Athenian Legacies, 128-156.

Ober, Josiah: Living Freely as a Slave of the Law: Why Socrates Lives in Athens, in: OBer, Athenian Legacies, 157-170.

Ober, Josiah: 'I Besieged That Man': Democracy's Revolutionary Start, In RaAflaub/Ober/Wallace (Hrsgg.), Origins, 2007, Kapitel 4, 83-104.

Osborne, Robin: Greece in the Making, 2. überarbeitete Auflage, Routledge, London, 2009.

Piepenbrink, Karen: Politische Ordnungskonzeptionen in der attischen Demokratie des vierten Jahrhunderts v. Chr: eine vergleichende Untersuchung zum philosophischen und rhetorischen Diskurs, Steiner (Historia Einzelschriften 154), Stuttgart, 2001.

RaAflaub, Kurt: Die Entdeckung der Freiheit. Zur historischen Semantik und Gesellschaftsgeschichte eines politischen Grundbegriffes der Griechen, Beck (Vestigia 37), München, 1985 (= The Discovery of Freedom in Ancient Greece, revised and updated edition, Chicago 2004).

RaAflaub, Kurt: Contemporary Perceptions of Democracy in Fifth-Century Athens, Classica et Mediaevalia 40 (1989), 33-70 (=K. RaAflaub / W.R. Connor / M.H. Hansen / B.S. Strauss: Aspects of Athenian Democracy, Museum Tusculanum Press, Copenhagen 1990, 33-70).

RaAflaub, Kurt: Einleitung und Bilanz: Kleisthenes, Ephialtes und die Begründung der Demokratie. In K. KInzl (Hg.), Demokratia: Der Weg zur Demokratie bei den Griechen, Wissenschaftliche Buchgesellschaft, Darmstadt, 1995, 1-54, 451-52 (=Wege der Forschung 657).

RaAflaub, Kurt: Legend or Historical Personality? Solon Reconsidered, In: J. Papademetriou (Hg.), Acta: First Panhellenic and International Conference on Ancient Greek Literature, Athens 1994, Hellenic Society for Humanistic Studies, Athens, 1997, 97-117. 
RaAflaub, Kurt: Poets, Lawgivers, and the Beginnings of Greek Political Reflection, in: Christopher Rowe / Malcolm Schofield (Hg.), The Cambridge History of Greek and Roman Political Thought, Cambridge University Press, Cambridge, 2000, 23-59.

RaAflaub, Kurt: Introduction. In: RaAflaub / OBer / Wallace 2007, 1-21.

RaAflaub, Kurt: The Breakthrough of Démokratia in Mid-Fifth-Century Athens, in: RaAflaub / Ober / Wallace 2007, 105-154.

RaAflaub, Kurt / Robert W. Wallace: "People's Power" and Egalitarian Trends in Archaic Greece, in: RaAflaub / Ober / Wallace 2007, 22-48.

Raaflaub, Kurt A./ Josiah Ober / Robert W. Wallace (Hgg.): Origins of Democracy in Ancient Greece. University of California Press, Berkeley, California 2007.

Raaflaub, Kurt A. / Hans Van Wees: A Companion to Archaic Greece, Wiley-Blackwell, Malden, MA., 2009.

Rhodes, Peter John: The Athenian Revolution, in: CAH V $\mathrm{V}^{2}$ (1992), 67-75.

Rhodes, Peter John (Hg.): Athenian Democracy, Edinburgh University Press, Edinburgh, 2004.

Rhodes, Peter John / Robin Osborne: Greek Historical Inscriptions, 404323 B.C., Oxford University Press, Oxford, 2003.

Robinson, Eric W:: The First Democracies. Early Popular Government Outside Athens, Steiner (Historia Einzelschriften 107), Stuttgart, 1997.

Robinson, E. W. (Hg.): Ancient Greek Democracy. Readings and Sources, Blackwell, Malden, MA; Oxford, 2004.

Rothwell, Kenneth Sprague: Politics and persuasion in Aristophanes' ecclesiazusae, Brill, Leiden, 1990.

Ruschenbusch, Eberhard: $\Sigma O \Lambda \Omega N O \Sigma$ NOMOI. Die Fragmente des solonischen Gesetzeswerkes mit einer Text- und Überlieferungsgeschichte, Steiner (Historia Einzelschriften 9), Wiesbaden, 1966.

RusChenbusCh, EBerhard: Zur Besatzung athenischer Trieren, Historia 28 (1979), 106-110.

Ruschenbusch, Eberhard: Die soziale Zusammensetzung des Rates der 500 in Athen im 4. Jh., ZPE 35 (1979), 177-180.

Sallares Robert: The Ecology of the Ancient Greek World, Duckworth, London, 1991.

Samons II, Loren J.: What's Wrong with Democracy. From Athenian Practice to American Worship, University pf California Press, Berkeley, California, 2004.

Schubert, Сh.: Der Areopag - Ein Gerichtshof zwischen Politik und Recht. In: L. Burckhardt / J. von Ungern-Sternberg (Hgg.): Große Prozesse im antiken Athen, Beck, München, 2000, 50-65; 258-262.

Schubert, Ch.: Der Areopag als Gerichtshof, ZRG (Röm. Abt.) 117 (2000), 103-132. 
Schumpeter, Joseph A.: Kapitalismus, Sozialismus und Demokratie, 8. Auflage, Francke (UTB), Tübingen; Stuttgart, 2005 (1. Auflage auf Englisch 1942).

Siewert, Peter (Hg.): Ostrakismos-Testimonien. Bd. 1: Die Zeugnisse antiker Autoren, die Inschriften und Ostraka über das athenische Scherbengericht aus vorhellenistischer Zeit (487-322 v. Chr.), Steiner, Stuttgart 2002 (=Historia-Einzelschriften 155).

Stahl, Michael: Gesellschaft und Staat bei den Griechen: Archaische Zeit, Schöningh (UTB), Paderborn, 2003.

Stahl, Michael: Antike und moderne Demokratie. Probleme und Zukunftsperspektiven der westlichen Demokratien im Spiegel des griechischen Bürgerstaates, in: WALTER EdER / KARL-JOACHIM HÖlKeSKAMP (Hgg.), Volk und Verfassung im vorhellenistischen Griechenland, Steiner, Stuttgart, 1997, 227-245.

Stahl, Michael / Uwe Walter: Athens, in: Raaflaub/Van Wees (Hgg.), Companion, 2009, 138-161.

Stone, I.F.: The Trial of Socrates, Cape, London, 1988.

Van Wees, Hans: Gute Ordnung ohne Große Rhetra. Noch einmal zu Tyrtaios Eunomia, in: Göttinger Forum für Altertumswissenschaft 5 (2002), 89-103. http://www.gfa.d-r.de/5-02/vanwees.pdf

Walter, Uwe: An der Polis teilnehmen: Bürgerstaat und Zugehörigkeit im archaischen Griechenland, Steiner, Stuttgart, 1993 (=Historia Einzelschriften 82).

Waterfield, Robin: Why Socrates Died: Dispelling the Myths, Faber, London, 2009.

Welwei, Karl-Wilhelm: Die griechische Polis: Verfassung und Gesellschaft in archaischer und klassischer Zeit, 2. Auflage, Steiner, Stuttgart, 1998.

Whitenead, David: The Demes of Attica, 508/7 - ca. 250 B.C., A Political and Social Study, Princeton University Press, Princeton, NJ, 1986.

Will, Wolfgang: Perikles, Rowohlt, Reinbek, 1995. 